\title{
Optimal Multi-Criteria Waypoint Selection for Autonomous Vehicle Navigation in Structured Environment
}

\author{
José Vilca • Lounis Adouane • Youcef Mezouar
}

Received: date / Accepted: date

\begin{abstract}
This paper deals with autonomous navigation of unmanned ground vehicles (UGV). The UGV has to reach its assigned final configuration in a structured environments (e.g. a warehouse or an urban environment), and to avoid colliding neither with the route boundaries nor any obstructing obstacles. In this paper, vehicle planning/set-points definition is addressed. A new efficient and flexible methodology for vehicle navigation throughout optimal and discrete selected waypoints is proposed. Combining multi-criteria optimization and expanding tree allows safe, smooth and fast vehicle overall navigation. This navigation through waypoints permits to avoid any path/trajectory planning which could be time consuming and complex, mainly in cluttered and dynamic environment. To evaluate the flexibility and the efficiency of the proposed methodology based on expanding tree (taking into account the vehicle model and uncertainties), an important part of this paper is dedicated to give an accurate comparison with another proposed optimization based on the commonly used grid map. A set of simulations, comparison with other methods and experiments, using an urban electric vehicle, are presented and demonstrate the reliability of our proposals.
\end{abstract}

Keywords Autonomous vehicle navigation · Optimal planning · Waypoints generation · Multi-criteria optimization · Vehicle's kinematic constraints . Localization under uncertainties.

José Vilca · Lounis Adouane · Youcef Mezouar Institut Pascal, Blaise Pascal University - UMR CNRS 6602, Clermont-Ferrand, France

Tel.: +33-47340-5532

Fax: +33-47340-7262

E-mail: Firstname.Lastname@univ-bpclermont.fr

\section{Introduction}

Fully autonomous vehicle navigation is a complex problem of major interest for the research community. Systems capable of performing efficient and robust autonomous navigation are unquestionably useful in many robotic applications such as manufacturing technologies [27], urban transportation [19], assistance to disabled or elderly people [25] and surveillance [30]. Even if a lot of progress has been made, some specific technologies have to be improved for real applications. This paper addresses specifically the problem of planning/set-points definition for autonomous navigation of vehicle in an urban environment (cf. Fig. 1).

\subsection{Overview of navigation strategies}

Different strategies for autonomous navigation have been proposed in the literature [2], [10] and [22]. The most popular approaches are based on the tracking of a predefined reference trajectory [24]. The reference trajec-

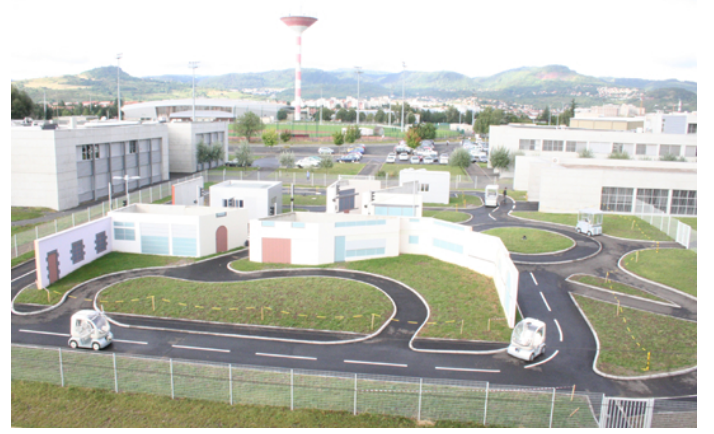

Fig. 1 Autonomous navigation of UGVs in a dedicated structured environment (Clermont-Ferrand, France). 
tory can be obtained using a combination of path planning and trajectory generation techniques [21]. The computation of a time-parameterized path while taking into account different vehicle constraints and environment characteristics is time-consuming [18] and [7]. Different algorithms for computation of a safe path (without temporal reference) require less computational time [21]. Typically, to obtain the reference path to be followed by the vehicle, arc-lines, B-splines or polynomial equations are used to interpolate points/waypoints [11], [8], [19] and [22]. A method to obtain these points was proposed in [28]. In [28], the authors use agent's observation and the geometric characteristics of the environment to select the waypoints. These waypoints can be used to reduce the planning time of existing planners. However, the method is based only on the position, the orientation and vehicle model are not taken into account. In [10], a feasible path is obtained using a polynomial curvature spiral. In [6], the trajectory generation method generates a smooth path considering the kinodynamic constraints of the vehicle. In [19], trajectories are built using user assigned points and interpolation functions such as cubic splines, trigonometric splines and clothoids. Moreover, velocity profiles along the trajectory are specified to improve the passengers comfort which is related to the acceleration. Nevertheless, trajectory generation presents some drawbacks such as the necessity of a specific planning method, a guarantee of continuity between different segments of the trajectory and more flexibility for dynamic replanning.

Otherwise, contrary to follow/track a trajectory to lead the robot toward its objective, few works in the literature propose to use only specific way-points in the environment to lead the robot toward its final objective. In [3], the authors propose a navigation via assigned static points for a unicycle robot. Nevertheless, the definition of the mission is less accurate because this strategy does not consider: the kinematic constraints of the robot (maximum velocity and steering), the orientation error and the velocity profile of the robot when it reaches the assigned point. In this paper, we present a navigation strategy which avoids the generation of a specific reference trajectory. Vehicle movements are generated using the control law that we have recently proposed in [33]. We will demonstrate in this paper that only few waypoints will be sufficient to guarantee safe and flexible vehicle navigation. We propose two methods to obtain these optimal waypoints in a known environment. These methods can take into account vehicle kinematic and perception/vehicle model uncertainties. The main advantage of this navigation strategy is its flexibility. The vehicle can perform different movements between waypoints without the necessity of replanning any reference trajectory, and it can also add or change the location of the successive waypoints according to the environment configuration or the task to achieve. This strategy allows thus flexible navigation while taking into account appropriate waypoints suitably placed in the environment.

\subsection{Related works}

Different algorithms can be used to obtain waypoints configuration such as $A^{*}, D^{*}[7]$, Rapidly Random Tree (RRT) [18], Sparse $A^{*}$ Search (SAS) [31]. At this aim, Configuration space (C-space), space of all possible configuration of the robot [29], enables the identification of the safe area where the vehicle can navigate without a collision risk (free space C-space ${ }_{\text {free }}$ ). C-space is used to compute the minimum distance to $\mathrm{C}$-space ${ }_{\text {obst }}$ (obstacle or road boundaries space). Fig. 2 shows the Cspace and its Voronoï diagram [20] in gray scale w.r.t. the distance to the closest C-space ${ }_{\text {obst }}$ (the whitest area represents the safest area).

Typically, algorithms based on grid map (e.g., $A^{*}$ or $D^{*}$ ) produce the shortest path by optimization of a criterion such as the distance to the goal, distance to the risk area, etc. [7]. The algorithm begins generally at the final cell (final position) and traverses the cell's neighbors until to reach the initial position. The cost of traveling through the neighbor is added to the total cost, the neighbor with the lowest total cost is selected, and so on. The process terminates once the initial position is reached. The path is given through the cell positions of the grid map while backtracking

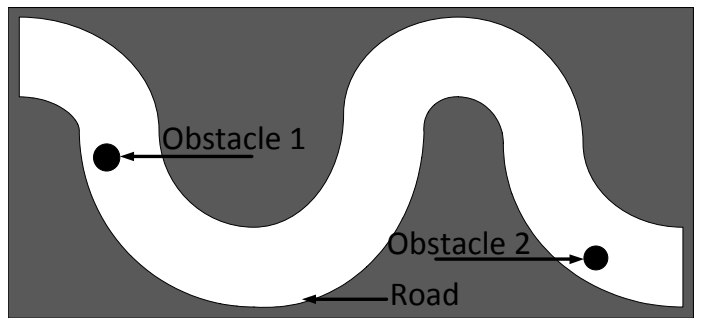

(a)

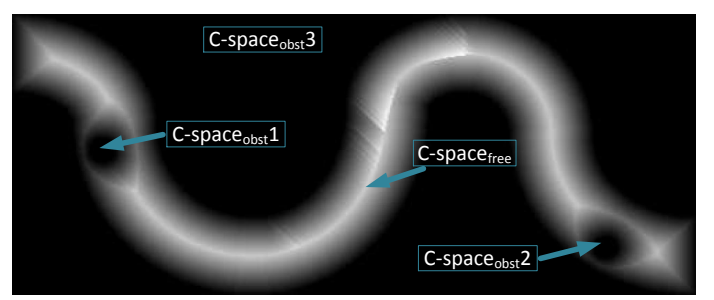

(b)

Fig. 2 (a) Road scheme and (b) its C-space with its Voronoï diagram. 
the cells which have the lower path cost, sometimes a polynomial interpolation is used to obtain a smooth path [8]. In [34], the authors present an $A^{*}$ algorithm using clothoid trajectories assuming constant velocity along them. Therefore, appropriate waypoints can be selected from this shortest path while only considering the cells where an orientation change occurs (w.r.t. its predecessor). Nonetheless, this algorithm does not consider neither former/initial vehicle orientation nor its kinematic constraints.

Instead of using grid map, it is possible also to obtain safe, feasible and smooth path using expanding tree algorithms (e.g., RRT, RRT* or SAS [21], [18], [15] and [31]). This could be done by providing to the vehicle-model the commands to reach the successive selected nodes until the goal [21], [18] and [31]. The basic process of RRT consists in selecting, at each sample time, a random node $q_{\text {random }}$ in the $\mathrm{C}$-space free $_{\text {r }}$ This selection considers generally only position $q_{\text {random }}=$ $\left(x_{\text {random }}, y_{\text {random }}\right)$ without any a priori vehicle orientation [21]. Then, the commands (discrete values) are applied to vehicle (from its current position and orientation) during a constant time $t_{\text {exp }}$. The vehicle model and constant commands allow to predict the final vehicle position at the end of $t_{\text {exp }}$. The commands that produce the closest position $q_{\text {chosen }}$ (a node which optimizes a dedicated task criterion [32]) to current random node $q_{\text {random }}$ are selected and stored with $q_{\text {choose }}$. A new expansion starts until to reach $q_{\text {random }}$ or to select a new random node $q_{\text {random }}^{\text {new }}$. Therefore, the waypoints can be selected, as in the case of grid map, while only considering the nodes where an orientation change occurs (w.r.t. its predecessor node). Algorithms based on RRT are very useful for motion planning because they can provide the commands (based on the kinematic/dynamic model of the vehicle) to reach the desired final configuration [18] and [32]. Moreover, it avoids the use of grid maps that can increase the computation time for large environment. In [31], the authors use the expanding tree for trajectory planning introducing different constraints such as maximum turning angle and route distance. Nevertheless, this method does not consider neither the vehicle movements along the trajectory nor localization uncertainties. In [14], sequential composition of controllers (e.g., go to the landmark and wall following controller) are used to generate valid vehicle states $q_{\text {choose }}$ to the navigation function. This approach avoids to find a single globally attractive control law and allows to use some additional sensing capability of the robot when the landmark is unreachable (e.g. GPS-denied area). However, the obtained navigation function has a complex computational processing. The most important drawbacks of expanding tree algorithms are the slow convergence to cover all space until to reach the goal and that in most cases it does not provide the shortest path since the nodes are randomly selected [1]. Furthermore, it is important to underline that in the RRT the control commands are maintained during a certain time, whereas in this paper the vehicle movement takes into account the definition of the used control law in addition to the vehicle model (cf. subsection 3.2). A comparison with RRT and Voronoï approaches is shown in subsection 4.1.2.

In this paper, we propose a method based on expanding tree to obtain the optimal waypoint configuration in a structured environment (cf. Fig. 4). It allows to consider constraints such as the kinematic model and the used control law. Criteria to optimize are related to the kinematic constraints of the vehicle (non-holonomy, maximum velocity and steering angle) and localization uncertainties. To highlight the advantages and flexibility of our proposal, a comparison with another proposed method, based on the commonly used grid map, is presented (cf. Section 3). The method based on grid map algorithm considers the vehicle as one cell without constraints and it can move only through the cells of the grid map. The trajectory of the vehicle depends on the choice of the waypoint configuration (cf. Section 4). We will show that the method based on grid map is less flexible and less efficient with regards to the methods based on expanding tree (cf. Section 4).

The rest of the paper is organized as follows: the next section presents the navigation framework, the waypoint assignment strategy for navigation, the vehicle model and its control law. The selection of waypoint configurations in the environment using a multi-criteria optimization techniques is described in Section 3. Simulation and experiments are given in Section 4. Finally, Section 5 provides a conclusion and prospects for future studies.

\section{Overall autonomous navigation framework}

An important condition in the field of autonomous vehicle is to ensure safe and flexible vehicle navigation (cf. Fig. 4). In this work, safe navigation consists in not colliding with the road limits and other obstacles while respecting the physical constraints of the vehicle. Flexible navigation consists in allowing different possible movements to achieve the task, while guaranteeing a smooth trajectory of the vehicle. Certainly, the main idea of the proposed work is to guarantee both criteria simultaneously.

This paper focuses on the method to obtain the optimal set of waypoints appropriately located in the en- 
vironment to perform a safe, flexible and fast vehicle navigation (cf. Section 3). Nevertheless, before detailing this multi-criteria optimization problem, let us first present in subsection 2.1 the details of the autonomous navigation strategy based on finite and sequential waypoints assignment described in [33].

\subsection{Navigation from sequential waypoint assignment}

A waypoint corresponds to a specific key configurations $\left(x_{q_{i}}, y_{q_{i}}, \theta_{q_{i}}, v_{q_{i}}\right)$ (where $\left(x_{q_{i}}, y_{q_{i}}\right)^{T}, \theta_{q_{i}}$ and $v_{q_{i}}$ denote to respectively the position, the orientation and the velocity of the waypoint $q_{i}$ ) in the environment as given in Fig. 3 (cf. Section 3). A vehicle navigation using only waypoints allows to avoid any path/trajectory planning which could be time-consuming and complex, mainly in cluttered and dynamic environment. Moreover, this kind of navigation does not require the pose of the closest point to any trajectory (w.r.t. the robot configuration) and/or the value of the curvature at this point [10]. Consequently, the navigation problem is simplified to a waypoint reaching problem, i.e, the UGV is guided by waypoints (cf. Fig. 3) instead of following a specific fixed path. Moreover, it is important to notice that if the successive waypoints are closer to each other then the vehicle tends to perform a path following navigation. To simplify the computation of the waypoints, they could be selected from a pre-defined path if it is available [33]. Indeed, a safe reference path can be obtained by different algorithms such as a Voronoï diagram [20] or potential fields [17]. Nevertheless, adding this step of path planning (with all its possible drawbacks (cf. section 4)) before obtaining the set of waypoints, restricts considerably the C-space free $_{\text {to only a }}$ curvilinear line. Thus, the optimality of the obtained set of Waypoints is not guaranteed (cf. Section 3).

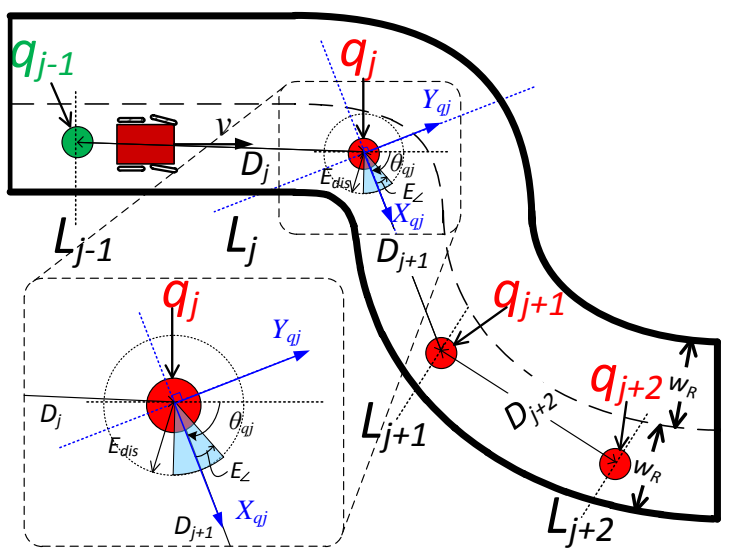

Fig. 3 Description of waypoints assignment.

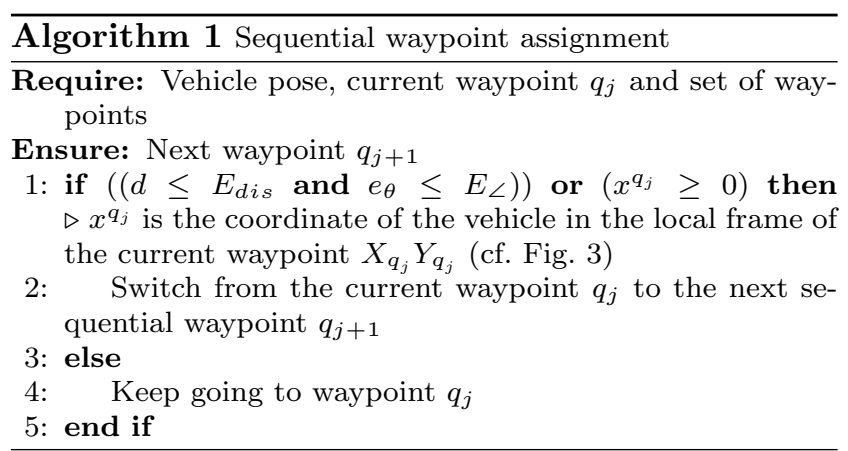

The strategy proposed in [33] uses a sequence of waypoints suitably positioned in the environment. To navigate between successive waypoints (e.g. $q_{j}$ and $q_{j+1}$ ), the distance of the vehicle to the target $d$ and the error $e_{\theta}$ between the orientation of the vehicle and the target are used. Their maximum values $\left(E_{d i s}\right.$ and $E_{\angle}$ respectively (cf. Fig. 3)) are imposed to the current waypoint $q_{j}$ to be reached. These values are notably related to the inaccuracies of the vehicle localization and/or the performance of the used control law. The maximum authorized values allow to keep a reliable vehicle control towards the target $T_{j}$ (cf. Fig. 3) while guaranteeing the appropriate vehicle configuration to reach the next target $T_{j+1}$.

Figure 3 shows a set of successive waypoints. $D_{j}$ is the Euclidean distance between the waypoints $q_{j-1}$ and $q_{j}$. For simplicity, the orientation of the waypoint $\theta_{q_{j}}$ is represented as the orientation of the line that joins $q_{j}$ and $q_{j+1}$. The strategy to assign at each sample time the appropriate waypoint is shown in Algorithm 1. The parameters of the control law enable the vehicle to reach the next waypoint (cf. Subsection 2.3) while ensuring that the vehicle trajectory is always within the road boundaries (cf. Section 4). The error conditions, $E_{\text {dis }}$ and $E_{L}$, are used to switch to the next waypoint when the vehicle position enters a circle with a radius equal to $E_{d i s}$ and center $\left(x_{q_{j}}, y_{q_{j}}\right)$. The current waypoint is updated with the following waypoint and the vehicle starts the movement to reach this new waypoint. If the vehicle does not satisfy the distance and orientation error conditions w.r.t. the current waypoint $q_{j}$ then the perpendicular line $L_{j}\left(Y_{q_{j}}\right.$ axis) to the road at the current waypoint is used to switch to the next waypoint when the vehicle crosses $L_{j}$ (cf. Fig. 3).

Before presenting briefly the control law to reach sequentially each single waypoint (cf. subsection 2.3), let us present the navigation scenario and the model of the vehicle used for the control law definition. 


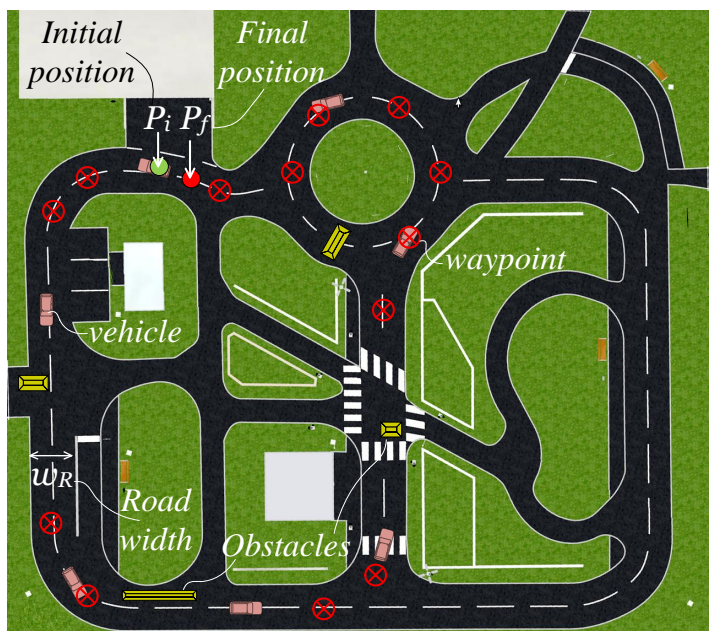

Fig. 4 Nominal scenario related to the effective platform (cf. Fig. 23) with the road map and the vehicle navigation.

\subsection{Navigation scenario and vehicle modeling}

The following scenario is considered (cf. Fig. 2 and 4):

- The environment of navigation is known throughout a map, containing the position of all static obstacles.

- The kinematic/dynamic model of the vehicle is known (with potentials uncertainties).

- The vehicle starts at the initial pose $P_{i}$ and it has to reach the final position $P_{f}$ (in certain cases, $P_{i}=$ $\left.P_{f}\right)$.

The UGV evolves in asphalt road and in cluttered urban environment with relatively low speed (less than $v_{\max }=3 \mathrm{~m} / \mathrm{s}$ ). Hence, the use of kinematic model of the UGV is sufficient (which relies on pure rolling without slipping). The kinematic model of the UGV is based on the tricycle model [23]. The two front wheels are replaced by a single virtual wheel located at the center between the front wheels (cf. Fig. 5). In [13], different tricycle designs are described giving the relation between the vehicle wheels velocities and the global vehicle kinematic model (linear and angular velocities). This relation is important to take into account for dynamic modeling of the vehicle [13]. Nevertheless, as mentioned above, the kinematic model is enough for our application. The kinematic model is given by (cf. Fig. 5):

$\left\{\begin{array}{l}\dot{x}=v \cos (\theta) \\ \dot{y}=v \sin (\theta) \\ \dot{\theta}=v \tan (\gamma) / l_{b}\end{array}\right.$

where $(x, y, \theta)$ is the vehicle pose in the global reference frame $X_{G} Y_{G} . v$ and $\gamma$ are respectively the linear velocity and orientation of the vehicle front wheel. $l_{b}$ is the wheelbase of the vehicle.

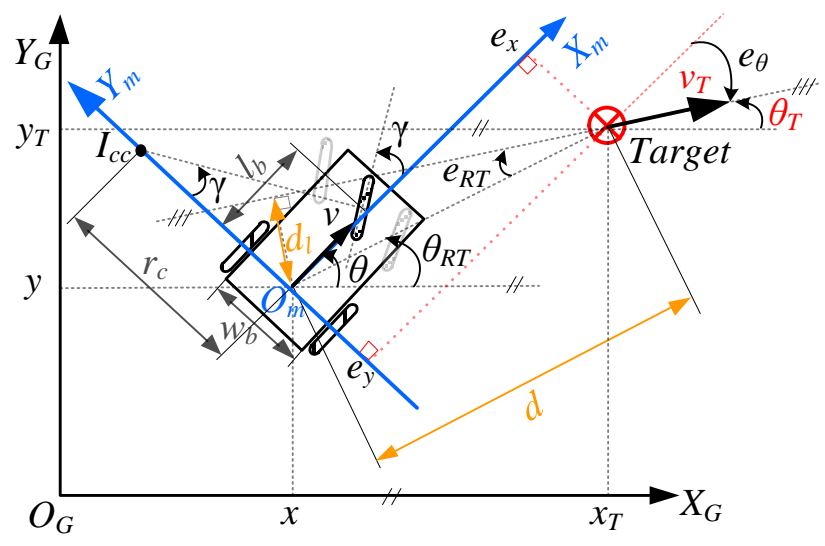

Fig. 5 UGV and target configuration variables in Cartesian references frames (local and global).

\subsection{Target reaching controller}

The target set-point modeling corresponds to a single waypoint configuration $\left(x_{T}, y_{T}, \theta_{T}, v_{T}\right)$, where $\left(x_{T}, y_{T}, \theta_{T}\right)$ and $v_{T}$ are respectively the pose and velocity of the target. For static target reaching, $v_{T} \neq 0$ is considered as a desired velocity value for the vehicle when it reaches the desired target pose.

The target reaching controller guides the vehicle sequentially towards the current assigned static target (cf. subsection 2.1). Before briefly describing the used control law [33], let us define the following notation (cf. Fig. 5):

$-O_{G}$ and $O_{m}$ are respectively the origin of global and local reference frames of the vehicle.

- $I_{c c}$ is the instantaneous center of curvature of the vehicle trajectory, $r_{c}=l_{b} / \tan (\gamma)$ is the radius of curvature and $c_{c}=1 / r_{c}$ is the curvature.

- $\left(e_{x}, e_{y}, e_{\theta}\right)$ are the errors w.r.t local frame $\left(X_{m} Y_{m}\right)$ between the vehicle and the target poses.

$-\theta_{R T}$ and $d$ are respectively the angle and distance between the target and vehicle positions.

$-e_{R T}$ is the error related to the vehicle position $(x, y)$ w.r.t the target orientation.

$-d_{l}$ is the distance from the vehicle to the target orientation line.

This controller is based on the pose control of the UGV w.r.t. the target. Let us first introduce the error variables $\left(e_{x}, e_{y}, e_{\theta}\right)$ (cf. Fig. 5) defined in the local reference frame $X_{m} Y_{m}$ :

$\left\{\begin{array}{l}e_{x}=\cos (\theta)\left(x_{T}-x\right)+\sin (\theta)\left(y_{T}-y\right) \\ e_{y}=-\sin (\theta)\left(x_{T}-x\right)+\cos (\theta)\left(y_{T}-y\right) \\ e_{\theta}=\theta_{T}-\theta\end{array}\right.$

The error function $e_{R T}$ is added to the canonical error system (2). The parameters $d$ and $\theta_{R T}$ can be 
written as (cf. Fig. 5):

$$
\begin{aligned}
& d=\sqrt{\left(x_{T}-x\right)^{2}+\left(y_{T}-y\right)^{2}} \\
& \begin{cases}\theta_{R T}=\arctan \left(\left(y_{T}-y\right) /\left(x_{T}-x\right)\right) & \text { if } d>\xi \\
\theta_{R T}=\theta_{T} & \text { if } d \leq \xi\end{cases}
\end{aligned}
$$

where $\xi$ is a small positive value $(\xi \approx 0)$. The error $e_{R T}$ is defined as (cf. Fig. 5):

$e_{R T}=\theta_{T}-\theta_{R T}$

It can be written as a function of $e_{x}, e_{y}$ and $e_{\theta}$ as:

$$
\begin{array}{r}
\tan \left(e_{R T}\right)=\tan \left(e_{\theta}-\left(\theta_{R T}-\theta\right)\right) \\
=\frac{e_{x} \tan \left(e_{\theta}\right)-e_{y}}{e_{x}+\tan \left(e_{\theta}\right) e_{y}}
\end{array}
$$

where $\tan \left(\theta_{R T}-\theta\right)=e_{y} / e_{x}$ (cf. Fig. 5).

Finally the pose errors and velocities $\left(e_{x}, e_{y}, e_{\theta}, v_{T}\right)$ are the input of the control law. The control law is obtained from Lyapunov stability analysis (cf. Appendix). It guarantees that the static or dynamic target will be reached [33]. The desired vehicle linear velocity $v$ and its front wheel orientation $\gamma$, that make the errors $\left(e_{x}, e_{y}, e_{\theta}\right)$ converge to zero, can be chosen as:

$v=v_{T} \cos \left(e_{\theta}\right)+v_{b}$

$\gamma=\arctan \left(l_{b} c_{c}\right)$

where $v_{b}$ and $c_{c}$ are given by:

$v_{b}=K_{x}\left(K_{d} e_{x}+K_{l} d \sin \left(e_{R T}\right) \sin \left(e_{\theta}\right)+K_{o} \sin \left(e_{\theta}\right) c_{c}\right)$

$$
\begin{aligned}
c_{c}= & \frac{1}{r_{c_{T}} \cos \left(e_{\theta}\right)}+\frac{d^{2} K_{l} \sin \left(e_{R T}\right) \cos \left(e_{R T}\right)}{r_{c_{T}} K_{o} \sin \left(e_{\theta}\right) \cos \left(e_{\theta}\right)}+K_{\theta} \tan \left(e_{\theta}\right) \\
& +\frac{K_{d} e_{y}-K_{l} d \sin \left(e_{R T}\right) \cos \left(e_{\theta}\right)}{K_{o} \cos \left(e_{\theta}\right)}+\frac{K_{R T} \sin ^{2}\left(e_{R T}\right)}{\sin \left(e_{\theta}\right) \cos \left(e_{\theta}\right)}
\end{aligned}
$$

$\mathbf{K}=\left(K_{d}, K_{l}, K_{o}, K_{x}, K_{R T}, K_{\theta}\right)$ is a vector of positive constants defined by the designer. $K_{d}, K_{l}$ and $K_{o}$ are respectively related to the desired convergence of the distance and lateral and angular errors w.r.t. the assigned target. $K_{x}, K_{R T}$ and $K_{\theta}$ are related to the maximum linear and angular vehicle velocities (more details are given in [33]).

\section{Optimal Multi-criteria Waypoint Selection (OMWS)}

This section is dedicated to the selection of the discrete waypoints in structured environment (cf. Figure 4) in order to perform safe and flexible vehicle navigation. The waypoints are obtained from an efficient and flexible methodology based on multi-criteria optimization using either grid map (cf. section 3.1) or expanding tree (cf. section 3.2).

In the both proposed OMWS (i.e., based on Grid Map (GM) and Expanding Tree (ET)), waypoints are selected considering safe position on the road, feasibility of trajectories (smooth changes between the successive points and vehicle constraints) and uncertainties.

The waypoints assignment strategies (cf. sections 3.1 and 3.2) are formulated as an optimization problem and solved using dynamic programming [4] (cf. Formulation 1).

Formulation 1 (Optimization problem) For each discrete state $x_{k} \in X$ where $X$ is a nonempty and finite state space. The objective is to obtain the sequence of states to reach the final state $x_{K}$ while minimizing the following cost function:

$C\left(x_{K}\right)=\sum_{k=1}^{K} g\left(\operatorname{Pred}_{x_{k}} \rightarrow x_{k}\right)+h\left(x_{K}\right)$

where Pred $x_{x_{k}}$ is the predecessor state of $x_{k} . g$ is the immediate traveling cost function to go from Pred Prk $_{x_{k}}$ to $x_{k} . h$ is the future traveling cost function (heuristic) to go from the current state to the final state $x_{K}$. When the current state is the final state $x_{K}$ then $h\left(x_{K}\right)$ is equal to zero. This function $h$ contributes to improve the convergence of the suboptimal solutions towards the global optimal one [5].

3.1 Optimal Multi-criteria Waypoint Selection based on Grid Map (OMWS-GM)

Before describing the proposed algorithm and the criterion to optimize, let us provide some useful definitions. A grid map corresponds to a limited environment area decomposed generally on square cells [7] (cf. Fig. 6 and 7). Each cell of the grid map can be an obstacle or a free space (cf. subsection 1.2 for the definition of C-space ${ }_{\text {obst }}$ and $\mathrm{C}$-space free $_{\text {}}$ ). The exterior limits of the $\mathrm{C}_{\text {-space }}$ free area are defined by the user, even for open environment (cf. Fig. 4). For simplification, the dimension of the cells in the grid map is chosen according to the vehicle dimension. Therefore, the vehicle is contained, at each 
sample time, in only one cell [7]. We consider the center of the cell $(i, j)$ as its position. Each cell $(i, j)$ is defined by the following parameters:

$-\bar{w}_{i j} \in[0,1]$ is related to the normalized distance $d_{i j_{-} \text {To_Obst }}$ to the closest $\mathrm{C}$-space ${ }_{\text {obst }}$, and it is given by:

$$
\bar{w}_{i j}=1-\frac{d_{i j \_T o \_O b s t}}{d_{\text {max_To_Obst }}}
$$

where $d_{\text {max_To_Obst }}$ is the maximum value among all $d_{\text {cell_To_Obst }}$ of all cells in the C-space free $_{\text {. As an }}$ example, Fig. 7 shows different distances of cells localized at $(a, b),(i, j)$ and $(m, n)$ to the $\mathrm{C}$-space obst $_{\text {. }}$. $d_{\text {max_To_Obst }}$ is equal, in this example, to the maximum distance $d_{m n_{-} \text {To_Obst }}$.

- $S$ is the cell state, which has three possible values, Init (Initialization), Open (when it is in the expansion queue) and Close (when it has already been expanded).

- A set of neighbors defined by:

$$
\mathbf{S}_{N}\left(\operatorname{cell}_{i j}\right)=\left\{\left(i \pm \Delta i_{N}, j \pm \Delta j_{N}\right) \mid\left(\Delta i_{N}, \Delta j_{N}\right) \neq(0,0)\right\}
$$

where $\Delta i_{N}, \Delta j_{N}=1 \ldots, N_{h} . N_{h}$ is the neighborhood value (cf. Fig. 8). Fig. 8(a) shows the case where $N_{h}=1$ (which implies 8 neighbor cells). Fig. 8 (b) shows a larger neighbor cells when $N_{h}=2(24$ neighbors).

- Pred $_{i j}$ is the neighbor cell of $i j$ which minimizes the total cost $C(i j)$ (cf. Fig. 6).

- $g\left(\right.$ Pred $\left._{i j} \rightarrow i j\right)$ is the traveling cost from the predecessor cell until the current cell $i j$.

$-h(i j)$ is the heuristic traveling cost from the current cell $i j$ to the final cell. As conventional, this cost depends on the euclidean distance from the cell $i j$ to the final cell.

The traveling cost function $g\left(\right.$ Pred $\left._{i j} \rightarrow i j\right)=g(m n \rightarrow$ $i j)$, from $m n$ to $i j$, is normalized $(g \in[0,1])$. It is also designed to take into account the variation of cell orientation (cf. Fig. 6). It allows to obtain an optimal path consisting of minimum number of straight lines. Therefore, a lowest possible number of waypoints in the safe area can be extracted from this optimal path. The cost function $g(m n \rightarrow i j)$ is given by:

$g(m n \rightarrow i j)=k_{g} \bar{w}_{i j}+\left(1-k_{g}\right) \frac{\left|\alpha_{i j}-\alpha_{m n}\right|}{2 \pi}$

where the first term of (14) is related to the safety of the obtained solution, and the real constant $k_{g} \in[0,1]$ is used to increase or to decrease the significance of this term. The second term of (14) is related to the smoothness of the obtained solution, i.e, the path has

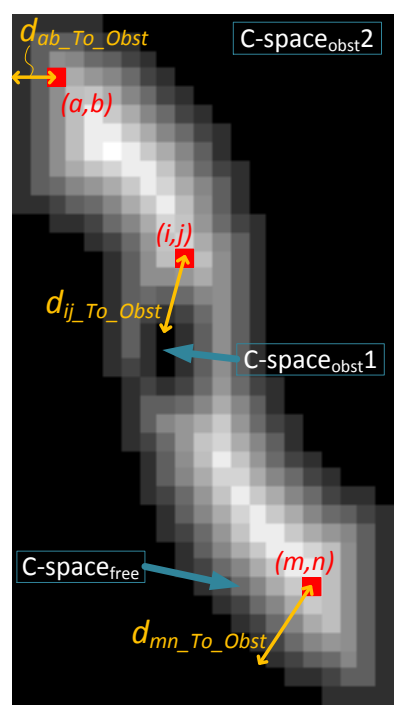

Fig. 7 Representation in gray scale w.r.t the distance to the closest C-space ${ }_{\text {obst }}$ (the whitest area represents the safest area).

a limited and minimum orientation change. The cell orientations $\left.\left.\alpha_{m n}, \alpha_{i j} \in\right]-\pi, \pi\right]$ are computed using the position of the current cell $(i, j)$, its predecessor $(m, n)$ and its probable successor $(a, b)$ (cf. Fig. 6). They are computed as:

$$
\begin{aligned}
\alpha_{i j} & =\arctan ([a-i] /[b-j]) \\
\alpha_{m n} & =\arctan ([i-m] / j-n])
\end{aligned}
$$

The heuristic traveling cost $h(i j) \in[0,1]$ (refers to (11)) is designed in function of the euclidean distance $d_{i j}$ from the cell $i j$ to the final cell. It is also used for the OMWS-ET (cf. subsection 3.2). The cost function $h(i j)$ is given by:

$h(i j)=k_{h}\left(1-e^{-d_{i j} / k_{e}}\right)$

where $k_{h} \in[0,1]$ allows to tune the significance of $h(i j)$ in the total cost function (11). The exponential function was chosen because it gives values between 0 and 1 for positive values of $d_{i j}$. The constant $k_{e} \in \mathbb{R}^{+}$is used to scale the value of $d_{i j}$ according to the dimensions of the environment. The value of $h(i j)$ (17) decreases while the next selected cell goes closer to the final cell.

Algorithm 2 shows in pseudocode, the first proposed method to obtain the set of waypoints in a structured and cluttered environment. It starts from the final vehicle position (initial cell). The algorithm selects the cells that have the lower total cost $C(i j)(11)$ until to reach the final cell. The set of waypoints is finally obtained, while tracking the predecessor cell of each selected cell which minimizes the total cost. 


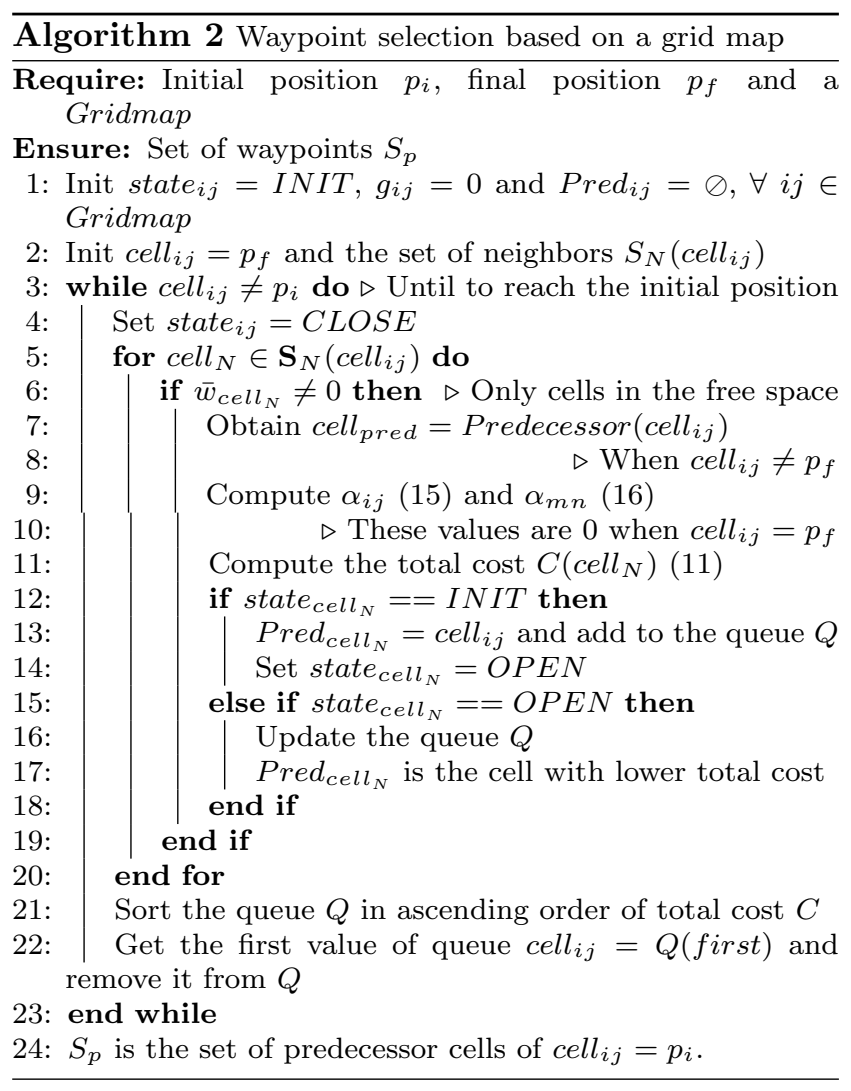

The obtained path is defined by straight lines connecting each two consecutive waypoints which belong to the set of obtained waypoints (cf. Algorithm 2). The smoothness of the path depends on the number of possible neighbors of the expanded cell defined by $N_{h}$ (cf. Fig. 9). The drawback of using a large number of neighbors is obviously the increasing of processing time. When $N_{h}>1$, it is mandatory to check if the current neighbor is blocked by some other forbidden neighbor (cf. Fig. 8(b)). For an off-line planning, $N_{h}>1$ can always be used to obtain a coherent and optimal solution regardless of time consumption.

3.2 Optimal Multi-criteria Waypoint Selection based on Expanding tree (OMWS-ET)

This subsection presents in details the main contribution of this paper for the optimal planning of the vehicle path, using an appropriate expanding tree. The formulation of this expanding tree integrates the kinematic model of the vehicle as well as the used control law definition and the vehicle localization uncertainties.

Before describing the proposed method and the criterion to be optimized, let us present the definition of expanding tree. The expanding tree is composed by nodes and edges which have the following properties:

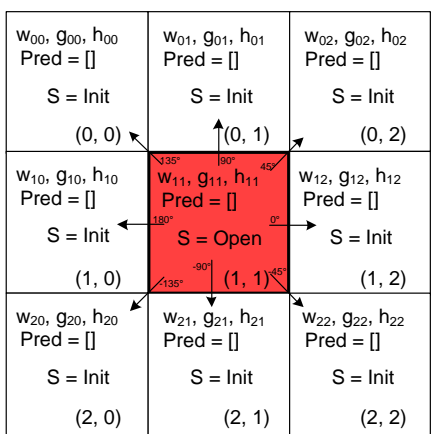

(a) $N_{h}=1$

\begin{tabular}{|c|c|c|c|c|}
\hline $\begin{array}{l}w_{00}, g_{00}, h_{00} \\
\text { Pred }=[]\end{array}$ & $\begin{array}{l}w_{01}, g_{01}, h_{01} \\
\text { Pred }=[]\end{array}$ & $\begin{array}{l}w_{02}, g_{02}, h_{02} \\
\text { Pred }=[]\end{array}$ & $\begin{array}{l}\mathrm{w}_{03}, \mathrm{~g}_{03}, \mathrm{~h}_{03} \\
\text { Pred }=[]\end{array}$ & $\begin{array}{l}\mathrm{w}_{04,} \mathrm{~g}_{04,}, \mathrm{~h}_{04} \\
\text { Pred }=[]\end{array}$ \\
\hline$S=$ Init & $S=$ Init & $S=$ Init & $S=$ Init & $S=$ Init \\
\hline$(0,0)$ & $(0,1)$ & $(0,2)$ & $(0,3)$ & $(0,4)$ \\
\hline $\begin{array}{l}\mathrm{W}_{10}, \mathrm{~g}_{10}, \mathrm{~h}_{10} \\
\text { Pred }=[]\end{array}$ & $\begin{array}{l}m_{11}, g_{11}, h_{11} \\
\text { Pred = [] }\end{array}$ & $\begin{array}{l}w_{12}, g_{11}, h_{12} \\
\text { Pred }=[]\end{array}$ & $\begin{array}{l}\text { w/13, } g_{13}, h_{13} \\
\text { Pred }=[\end{array}$ & $\begin{array}{l}\mathrm{w}_{14}, \mathrm{~g}_{14}, \mathrm{~h}_{14} \\
\text { Pred }=[]\end{array}$ \\
\hline$S=$ Init & $S=$ hxit & $S=$ Init & $s=$ Init & $\Rightarrow S=$ Init \\
\hline$(1,0)$ & 1 & $(1,2)$ & $(1,3)$ & $(1,4)$ \\
\hline $\begin{array}{l}\mathrm{W}_{20}, \mathrm{~g}_{20}, \mathrm{~h}_{20} \\
\text { Pred }=[]\end{array}$ & 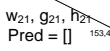 & 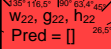 & $\begin{array}{l}W_{23}, g_{23}, \mathrm{~h}_{23} \\
\operatorname{Pred}=[]\end{array}$ & $\begin{array}{l}\mathrm{w}_{24}, \mathrm{~g}_{24}, \mathrm{~h}_{24} \\
\text { Pred }=[]\end{array}$ \\
\hline S = Init & $S=\operatorname{lnit} \leftarrow$ & $\mathrm{S}=\mathrm{Open}_{26, \mathrm{~s}}$ & $\rightarrow \quad \mathrm{S}=$ Init & $\longrightarrow \mathrm{S}=$ Init \\
\hline$(2,0)$ & $(2,1)$ & & $(2,3)$ & $(2,4)$ \\
\hline $\begin{array}{l}\mathrm{w}_{30}, \mathrm{~g}_{30}, \mathrm{~h}_{30} \\
\text { Pred }=[]\end{array}$ & $\begin{array}{l}W \\
\text { Pred }=g_{31}=0\end{array}$ & $\begin{array}{l}w_{32}, g_{22}, h_{3} \\
\text { Pred }=[]\end{array}$ & $\begin{array}{l}y_{13,}, g_{33}, h_{33} \\
\operatorname{Pred}=[]\end{array}$ & $\begin{array}{l}\mathrm{w}_{34}, \mathrm{~g}_{34}, \mathrm{~h}_{34} \\
\text { Pred }=[]\end{array}$ \\
\hline$S=\operatorname{lnit}$ & & $S=$ Init & $S=$ hit & $S=\operatorname{lnit}$ \\
\hline$(3,0)$ & $(3,1)$ & $(3,2)$ & $(3,3)$ & $(3,4)$ \\
\hline $\begin{array}{l}\mathrm{w}_{40}, \mathrm{~g}_{40}, \mathrm{~h}_{40} \\
\text { Pred }=[]\end{array}$ & 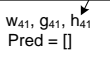 & $\begin{array}{l}\mathrm{w}_{42}, \mathrm{~g}_{42}, \mathrm{~h}_{42} \\
\text { Pred }=[]\end{array}$ & $\begin{array}{l}\mathrm{w}_{43}, \mathrm{~g}_{43}, \mathrm{~h}_{43} \\
\text { Pred }=[]\end{array}$ & $\begin{array}{l}\mathrm{w}_{44}, \mathrm{~g}_{44}, \mathrm{~h}_{44} \\
\text { Pred }=[]\end{array}$ \\
\hline$S=$ Init & $S=$ Init & $S=$ Init & $S=$ Init & $S=\operatorname{lnit}$ \\
\hline$(4,0)$ & $(4,1)$ & $(4,2)$ & $(4,3)$ & $(4,4)$ \\
\hline
\end{tabular}

(b) $N_{h}=2$.

Fig. 8 Different neighborhood values $N_{h}$ of the current cell (red). A gray cell represents a cell where the movements are forbidden.

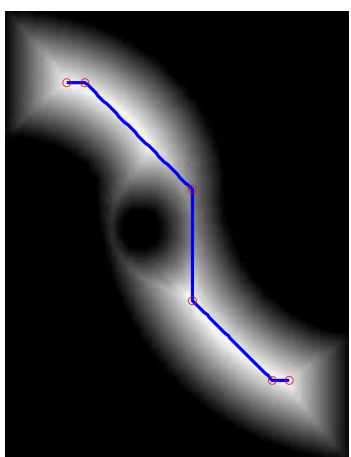

(a) $N_{h}=1$.

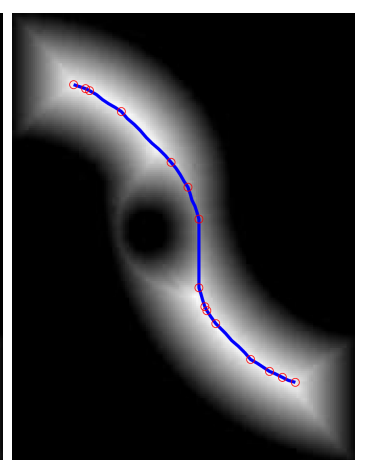

(b) $N_{h}=3$.
Fig. 9 Different sets of waypoints for different number of possible neighbor cells.

- Each node $q_{j}$ is defined by its pose $\left(x_{q_{j}}, y_{q_{j}}, \theta_{q_{j}}\right)^{T}$, one predecessor node $q_{i}$ (except for the initial node) and a traveling cost values $g\left(q_{j}\right)$ and $h\left(q_{j}\right)$ (cf. eq. (11)).

- Each edge $\xi_{i j}$ corresponds to the link between $q_{i}$ to $q_{j}$ nodes. 
- $g\left(q_{i} \rightarrow q_{j}\right)=g\left(\xi_{i j}\right)$ is the traveling cost from $q_{i}$ to $q_{j}$.

- $h\left(q_{j}\right)$ is the heuristic traveling cost from the current node $q_{j}$ to the final node (final vehicle pose). It is defined by (17) (cf. Subsection 3.1).

The traveling cost $g\left(\xi_{i j}\right) \in[0,1]$ is designed to obtain an appropriate balance among safe, smooth, feasible and fast trajectory of the vehicle. It is defined as:

$g\left(\xi_{i j}\right)=k_{1} \bar{w}_{j}+k_{2} \Delta \bar{v}_{i j}+k_{3} \Delta \bar{\gamma}_{i j}+k_{4} \Delta \bar{e}_{l_{i j}}$

where $k_{1}, k_{2}, k_{3}$ and $k_{4} \in \mathbb{R}^{+}$are constants which are defined by the designer to give the right balance (according to context of navigation, e.g., focus more on the safety with regard to the smoothness) of each term of the criterion (18). To normalize the traveling cost, $k_{i} \mid i=1, \ldots, 4$ must satisfy:

$k_{1}+k_{2}+k_{3}+k_{4}=1$

The normalization of the individual criterion given in (18) allows to simplify the choice of $k_{i}$ to select the priority of a term w.r.t. the others according to the navigation context. In Section 4, different set of values $k_{1}, k_{2}, k_{3}$ and $k_{4}$ will be considered for different scenarios.

The first term of the cost function (18) is related to the safety of the navigation (12). The second and third terms are respectively related to the speed (20) and smoothness (23) of the trajectory. The fourth term is related to feasibility of the vehicle trajectory while considering localization uncertainties, i.e., the risk to collide with an obstacle while considering inaccuracies in the vehicle position and orientation (a detailed explanation of this term is given later in this subsection). This last term allows to consider the kinematic model of the vehicle and the control law. The details of each term is given in the following:

- The term $\bar{w}_{j} \in[0,1]$ is related to the distance from the node $q_{j}$ to the closest $\mathrm{C}$-space ${ }_{\text {obst }}$. It is given by (12) (cf. Subsection 3.1).

- The term $\Delta \bar{v}_{i j} \in[0,1]$ is related to the velocity from $q_{i}$ to $q_{j}, v_{i j}$. It is given by:

$$
\Delta \bar{v}_{i j}=1-\frac{v_{i j}}{v_{\max }}
$$

where $v_{\max }$ is the maximum velocity of the vehicle. We estimate $v_{i j}$ as a function of the curvature of the trajectory. The maximum velocity occurs when the curvature is zero (straight line) and the minimum velocity $v_{\min } \neq 0$ occurs when the curvature is bigger than the value corresponding to $\gamma_{\max }$ (cf. Fig. 5). This consideration allows the vehicle maneuvers without risk of collisions while enhancing the passenger comfort [19] (indeed, this permits to limit the centripetal forces). The minimum and maximum values of velocity and steering angle are defined by the designer according to the vehicle characteristics. The curvature is estimated using the orientation of the current node and its predecessor. Therefore, $v_{i j}$ is computed as:

$v_{i j}=v_{\max }-\Delta \bar{\theta}_{i j}\left(v_{\max }-v_{\min }\right)$

where $\Delta \bar{\theta}_{i j} \in[0,1]$ is the normalized estimated curvature related to the variation of orientation between the current node $q_{j}$ and its predecessor $q_{i}$. It is defined as:

$\Delta \bar{\theta}_{i j}=\frac{\left|\theta_{j}-\theta_{i}\right|}{\Delta \theta_{\max }}$

where $\Delta \theta_{\max }$ is the maximum variation between a probable orientation of the current node w.r.t the orientation of its predecessor. This value is defined according to the steering capability of the vehicle. $\theta_{j}$ and $\theta_{i}$ are computed using the node positions and eq. (15) and (16) (cf. Subsection 3.1).

- The term $\Delta \bar{\gamma}_{i j} \in[0,1]$ is related to the variation of steering angle along the vehicle trajectory from $q_{i}$ to $q_{j}$ (for instance, Fig. 10 shows a vehicle trajectory between two nodes). It is given by:

$\Delta \bar{\gamma}_{i j}=\frac{\sum_{q_{i}}^{q_{j}}\left|\Delta \gamma_{i j}\right|}{n_{q_{i j}} \gamma_{\max }}$

where $n_{q_{i j}}$ is the considered point number of the vehicle trajectory between $q_{i}$ and $q_{j}$, and $\gamma_{\max }$ is the maximum steering angle of the vehicle. This term $\Delta \bar{\gamma}_{i j}(23)$ computes the sum of the $\Delta \gamma_{i j}$ to obtain the total variation of the steering angle along the vehicle trajectory. $\Delta \bar{\gamma}_{i j}$ uses the kinematic model and the control law to estimate the vehicle trajectory and commands or control set-points.

- The term $\Delta \bar{e}_{l_{i j}} \in[0,1]$ is the normalized maximum deviation of vehicle trajectory w.r.t. the straight line that joins the positions $\left(x_{q}, y_{q}\right)$ of $q_{i}$ and $q_{j}$ (cf. Fig. 10). It is computed as:

$\Delta \bar{e}_{l_{i j}}=\frac{\left|\Delta e_{l_{i j}}\right|}{\max \left\{\Delta e_{l}\right\}}$

where $\max \left\{\Delta e_{l}\right\}$ is the maximum deviation of all trajectories from the node $q_{i}$ to the node $q_{j}$ while considering the position and orientation uncertainties $\left(\epsilon_{d}\right.$ and $\epsilon_{\theta}$ respectively given in Fig. 10). This term $\Delta \bar{e}_{l_{i} j}$ allows to estimate the collision risk using the vehicle trajectory that takes into account the kinematic model, the control law and localization uncertainties (position and orientation). Fig. 


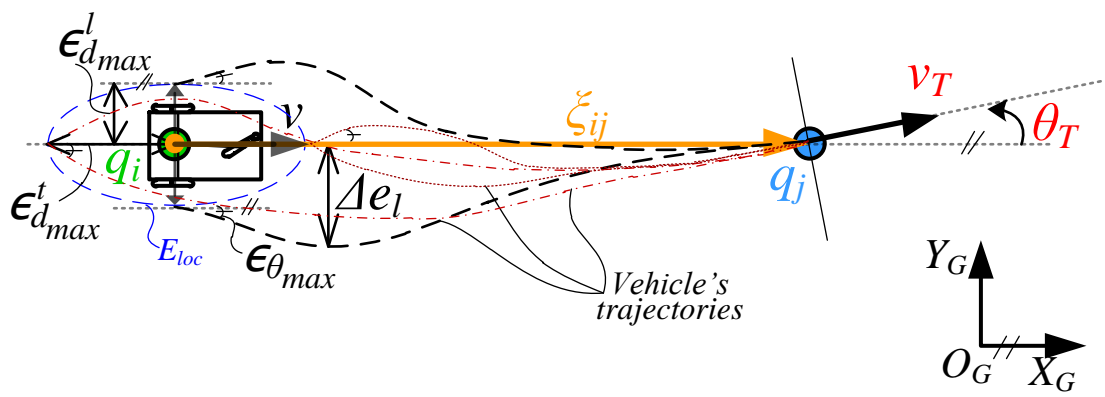

Fig. 10 Vehicle's trajectories which starts from extreme configurations $\left( \pm \epsilon_{d_{\max }}^{l}\right.$, $\pm \epsilon_{d_{\max }}^{t}$ and $\left.\pm \epsilon_{\theta_{\max }}\right)$ in the localization uncertainties ellipse $E_{l o c} . \Delta e_{l}$ is the maximum lateral deviation of all vehicle trajectories.

10 shows an illustration where the vehicle has an ellipse of localization uncertainties with axes $\epsilon_{d}^{l}$ and $\epsilon_{d}^{t}$. The vehicle trajectories start at $\pm \epsilon_{d}^{l}$ in lateral distance (longitudinal distance is set to 0 ), and $\pm \epsilon_{d}^{t}$ in longitudinal distance (lateral distance is set to 0 ) from the vehicle position with a $\pm \epsilon_{\theta}$ from the vehicle presumed orientation, i.e., we consider all extreme configurations to obtain, according to these maximum error configurations, the maximum lateral deviation $\left(\Delta e_{l}\right)$. The trajectories are obtained using the kinematic model and the used control law in an offline simulated procedure.

Algorithm 3 shows in pseudocode, the proposed method which uses expanding tree to obtain the optimal waypoints configurations w.r.t. the optimized multi-criteria function (18). Fig. 11 shows the first steps of the algorithm where, for instance, the branch numbers of each

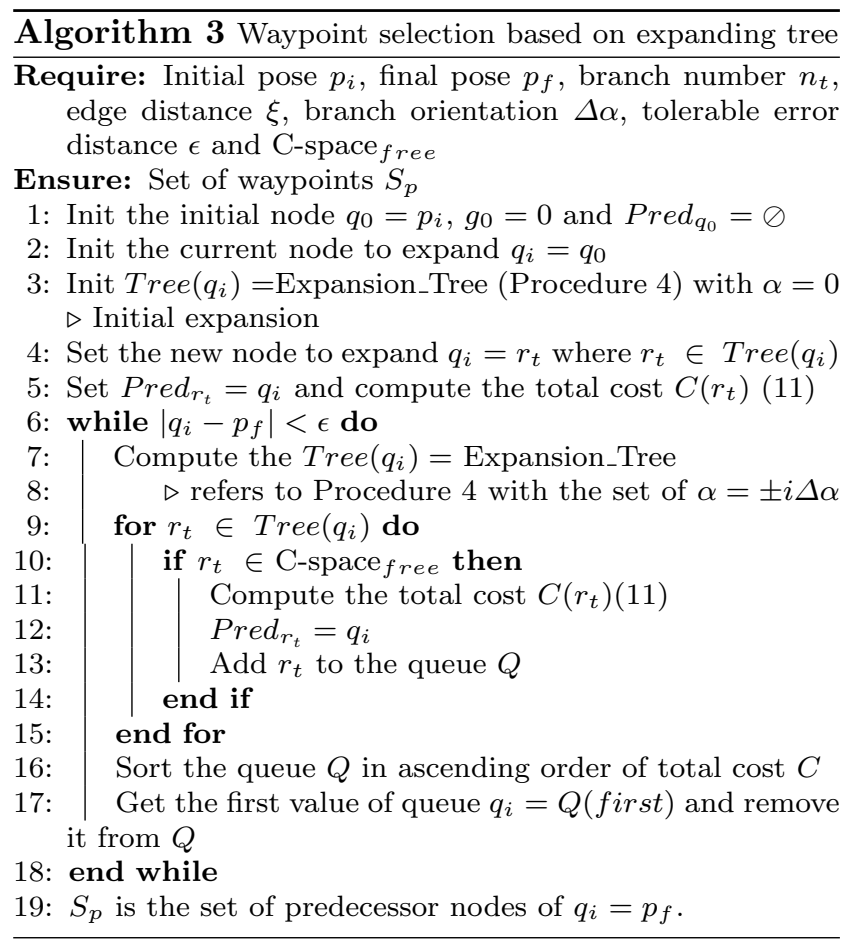

node is $n_{t}=3$ and each branch orientation w.r.t. the vehicle orientation is given by:

$\alpha= \pm i \Delta \alpha, \quad i= \begin{cases}0,1, \ldots,\left(n_{t}-1\right) / 2 ; & \text { if } n_{t} \text { is odd } \\ 1,2, \ldots, n_{t} / 2 ; & \text { if } n_{t} \text { is even }\end{cases}$

where $\Delta \alpha$ is a constant angle defined according to the vehicle characteristics.

The edge distance $\xi$ is the Euclidean distance between two successive nodes and it depends on the environment dimensions, e.g., if the environment has a narrow passage then $\xi$ must cope with this dimension. We consider that the edge orientation is the vehicle orientation at the current node position (cf. Fig. 11). Thus, at beginning the first expansion of $q_{0}$ is given with $\alpha=0$ because the vehicle starts at initial fixed pose (cf. line 3-5 of Algorithm 3). This initial expansion is made to respect the kinematic constraints where the rotation of the vehicle requires a displacement (linear velocity $\neq 0$ ) of the vehicle. Therefore, the successive node $q_{1}$ has different possible orientation and so on (cf. Fig. 11). The algorithm selects the node which has the lower total cost $C\left(q_{j}\right)(11)$. When two or more nodes have the same cost, the algorithm selects the last saved node. Fig. 11 shows the successive steps, the node $q_{2}$ was selected from the expansion of $q_{1}\left\{q_{2}, q_{3}, q_{4}\right\}$, which has the lower total cost value. The set of waypoints is obtained while tracking the predecessor nodes of the nodes with lower total cost. The selection of the node with lower total cost (cf. Algorithm 3, line 16-17) al-

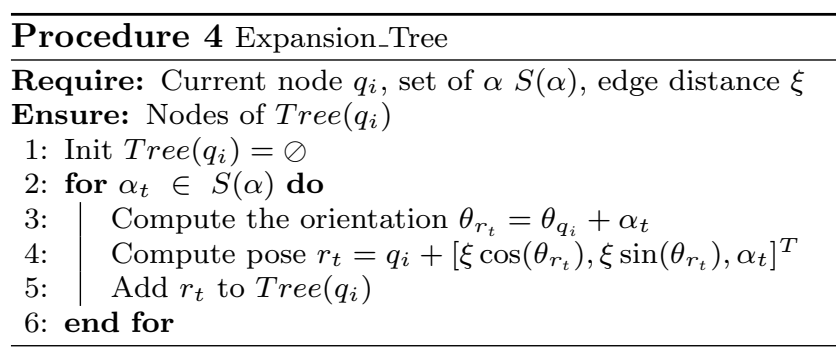




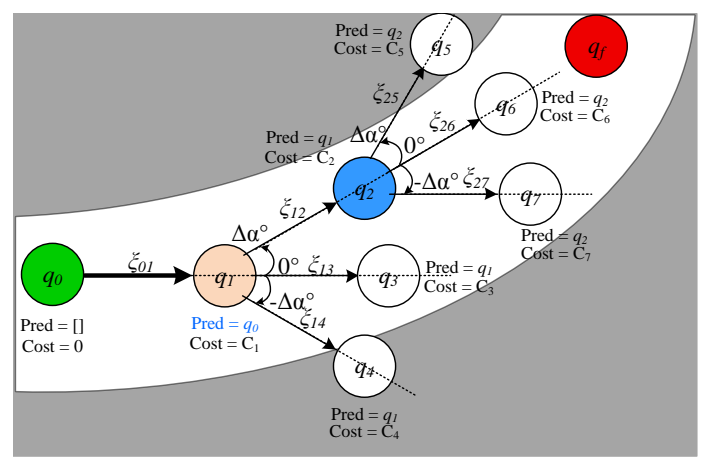

Fig. 11 Expanding tree method to obtain the appropriate set of waypoints.

lows to avoid the deadlock areas because the successive branches from the nodes in this deadlock area will be in C-space ${ }_{\text {obst }}$ (cf. Fig. 12(a)).

The smoothness of the vehicle trajectory depends on number of branches of each tree $n_{t}$, maximum branch orientation $\alpha_{\max }=n_{t} \Delta \alpha / 2$ and edge distance $\xi$ (cf. Section 4). The drawback of using a large number of $n_{t}$ is the increasing of the processing time required to obtain the set of waypoints. The vertex distance $\xi$ is set to detect obstacles between the successive nodes.

This method uses deterministic selection of expanding tree to obtain the optimal solution with lowest total cost. Nevertheless, a feasible solution can be obtained using a probabilistic selection of expanding tree to decrease the processing time (cf. Section 4), i.e., the branch orientation $\alpha$ and edge distance $\xi$ are randomly selected in a fixed interval [21]. In simulation, we will show in subsection 4.1 the case where these parameters are randomly chosen.

As described above, the traveling cost (18) depends on four parameters $\left(k_{i} \mid i=1, \ldots, 4\right.$, which satisfy (19)) related respectively to the safety, velocity, less steering and taking into account uncertainties. The values of these parameters are fixed according to the desired navigation and environment conditions. A pragmatical procedure to set these parameters consists in first identifying the main desired vehicle behavior and setting its parameter $k_{i}$ with a value greater than 0.5 (cf. Fig. 12). The other parameters will be tuned according to the designers secondary priorities. Fig. 12 shows the set of waypoints when only the term with highest priority is considered in the traveling cost function. For instance, in Fig. 12(a) and 12(b) the priority is given respectively to the safest and the shortest paths. More examples of different tuned parameters will be shown in subsection 4.1 .

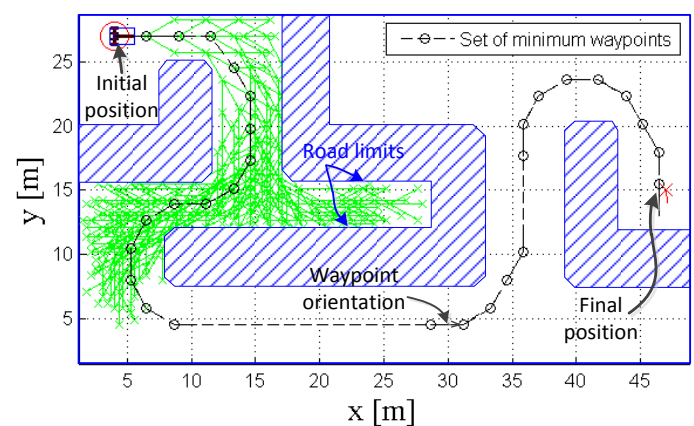

(a) Safest path: $k_{1}=1.0, k_{2}=k_{3}=k_{4}=0.0$ and $k_{h}=0.1$

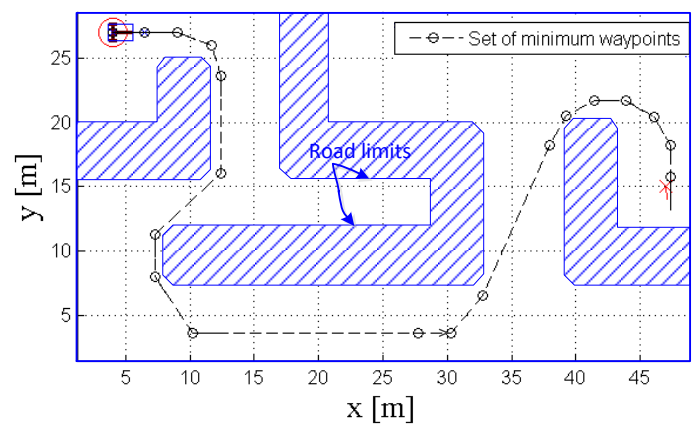

(b) Shortest path: $k_{2}=1.0, k_{1}=k_{3}=k_{4}=0.0$ and $k_{h}=0.1$

Fig. 12 Set of waypoints for different parameters values $k_{i}$ of the traveling cost.

\subsection{Minimum set of waypoints}

Algorithm 2 and 3 were applied to obtain a set of waypoints $S_{p}$ characterized by $\left(x_{q_{j}}, y_{q_{j}}, \theta_{q_{j}}, v_{q_{j}}\right)$ on a specific environment. The proposed Algorithm 5 will allow to reduce the number of waypoints. Basically, this algorithm keeps only the waypoints (its pose, velocity and predecessor are stored) which have an orientation changes w.r.t. its predecessors.

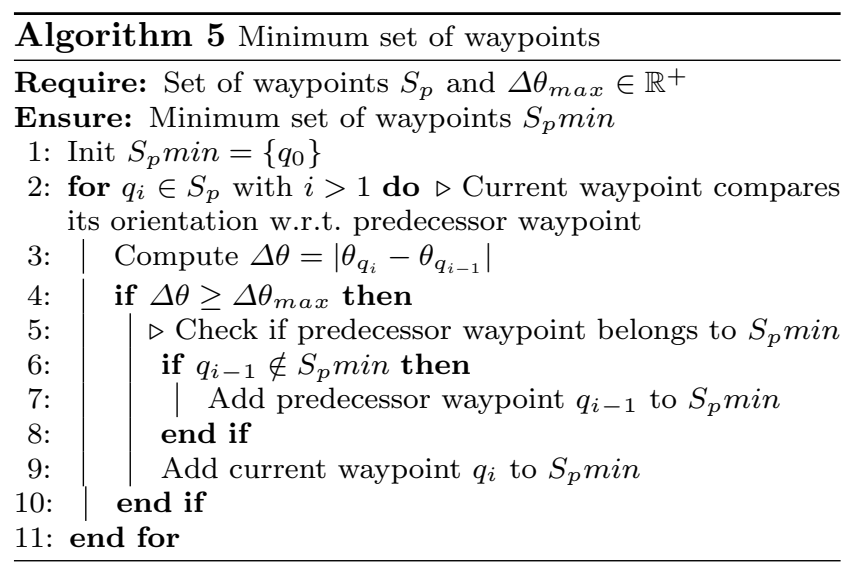




\section{Validation}

This section presents a set of experiments to demonstrate the efficiency of our methods for autonomous navigation in a structured environment. Section 4.1 provides different scenarios to show the validity of our proposals. Section 4.2 discusses experimental results applied to an urban electric vehicle.

\subsection{Simulations results}

This section shows optimal sets of waypoints, obtained according to the environment characteristics and/or the task to achieve. In what follows, it will be shown: a comparison between grid map and expanding tree algorithms (cf. subsection 4.1.1); a comparison between the proposed OMWS-ET and a variation of RRT (cf. subsection 4.1.2); different specific scenarios such as trajectory generation (cf. subsection 4.1.3); a comparison between deterministic and probabilistic waypoints selection (cf. subsection 4.1.4); an application of the proposed methodology of waypoints selection for multirobot formation (cf. subsection 4.1.5) and finally, subsection 4.1.6 shows the flexibility of our proposal for local replanning of the waypoints configurations when unexpected obstacles are detected. For these simulations, the physical parameters of the UGV are based on the urban vehicle VIPALAB (cf. Fig. 22) which is modeled as a tricycle (1). Its dimensions are $1.27 \mathrm{~m}$ (width), $1.96 \mathrm{~m}$ (length) and $2.11 \mathrm{~m}$ (height). The UGV constraints are minimum movement velocity $v_{\min }=$ $0.1 \mathrm{~m} / \mathrm{s}$, maximum velocity $1.5 \mathrm{~m} / \mathrm{s}$, maximum steering angle $\gamma_{\max }= \pm 30^{\circ}$ and maximum linear acceleration $1.0 \mathrm{~m} / \mathrm{s}^{2}$. The controller parameters are set to $\mathbf{K}=(1,2.2,8,0.1,0.01,0.6)$ (cf. subsection 2.3). These parameters were chosen to obtain a good balance between: accurate and fast response and smooth trajectory while taking into account the limits of the vehicle structural capacities. We consider that the sample time is $0.01 s$ and a maximum number of iteration is $n_{I}=5000$ to stop both algorithms, OMWS-GM (Algorithm 2) and OMWS-ET (Algorithm 3), when none solution can be obtained.

\section{1 .1 Grid map versus Expanding tree}

These simulations show two set of waypoints obtained by the two proposed methods based on grid map and expanding tree (cf. Algorithms 2 and 3 respectively). Fig. 13(a) and Fig. 14(a) show the set of obtained waypoints according to Algorithm 2 and 3. The minimum set of waypoints, obtained according to Algorithm 5, are given afterward in Fig. 13(b) and Fig. 14(b).
For the grid map case, the cell has the vehicle dimension $(2 \mathrm{~m})$ and its neighborhood is $N_{h}=1$. The constant value of $k_{g}$ is $0.6(14)$ and $k_{h}$ is 0.1 (17). The minimum set of obtained waypoints has $n_{w}=27$ elements. An additional constraint is considered for OMWS-GM (before line 11 of the Algorithm 2), the angle variation (second term of eq. (14) must be less than a threshold $\left.\theta_{t h}\right)$. This constraint enables the processing time of the algorithm to be reduced since it considers only the cells with an orientation change, w.r.t the last cell orientation, less than $\theta_{t h}$ (cf. Fig. 6).

For Expanding Tree case, the branch number $n_{t}$ is 5 , the edge distance $\xi$ is $2.5 \mathrm{~m}$ and $\Delta \alpha$ is $15^{\circ}$. We consider the safety gain $k_{1}$ (cf. eq. (18)) as the highest priority in this simulation. The constant values of $k_{i} \mid i=1, \ldots, 4$ (18) are $k_{1}=0.6, k_{2}=0.2, k_{3}=0.1, k_{4}=0.1$ and $k_{h}=0.1$ (17). The minimum set of obtained waypoints has $n_{w}=19$ elements. The minimum set of waypoint obtained by OMWS-ET is smaller than the method OMWS-GM which does not consider the orientation neither the vehicle's model. To avoid a large growing of the tree branch of OMWS-ET, a position and orientation comparison between nodes can be added at line 13 of the Algorithm 3. If two nodes from different branches have the same position and orientation then the node with lowest total cost function value (cf. eq. (11)) is stored and the other node is removed.

Table 1 shows different performance criteria to compare the set of waypoints where: $n_{w}$ is the number of waypoints, length is the sum of distance between two successive waypoints, $d_{\text {border }}$ is the sum of minimum distance to the road boundaries. Therefore, the method based on OMWS-ET is more safe, accurate and efficient than the one based on OMWS-GM, mainly when the criterion to optimize is related to the vehicle's model (velocity and steering angle).

\subsubsection{OMWS-ET versus $R R T^{*}$}

To highlight the advantages and the flexibility of the proposed OMWS-ET, a comparison with the popular RRT $^{*}$ algorithm [15] is presented in this subsection. The RRT* is based on the RRT (Rapidly-exploring Random Tree) already described in Section 1.2 with an addition of the rewiring function which allows to reconnect the nodes to ensure that the edges have the path with minimum total cost. RRT $^{*}$ provides thus an op-

\begin{tabular}{|c|c|c|c|}
\cline { 2 - 4 } \multicolumn{1}{c|}{} & $n_{w}$ & length $[\mathrm{m}]$ & $d_{\text {border }}[\mathrm{m}]$ \\
\hline OMWS-GM & 27 & 77.22 & 52.1268 \\
\hline OMWS-ET & 19 & 77.50 & 56.7217 \\
\hline
\end{tabular}

Table 1 Comparison between the OMWS-GM and ET. 


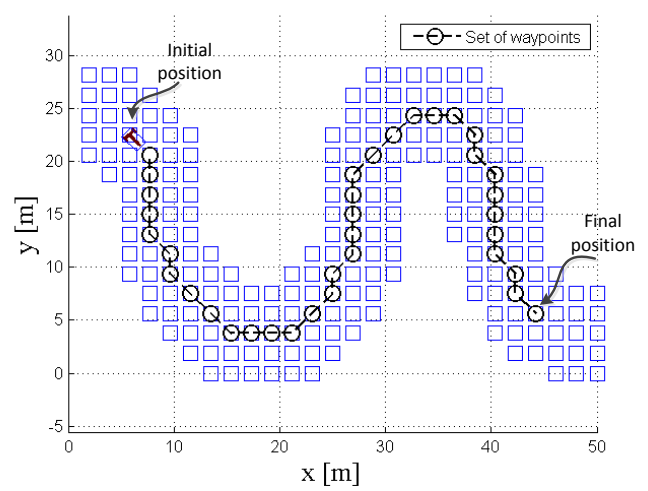

(a)

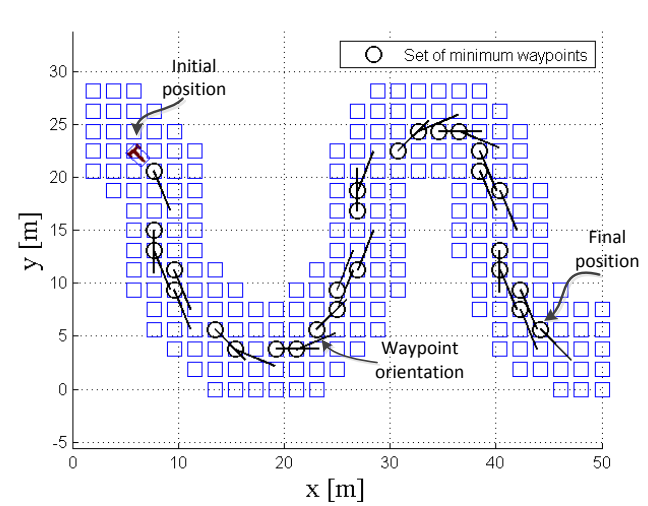

(b)

Fig. 13 a) Set of obtained waypoints using Algorithm 2 based on grid map and b) Minimum set of waypoints obtained by Algorithm 5.

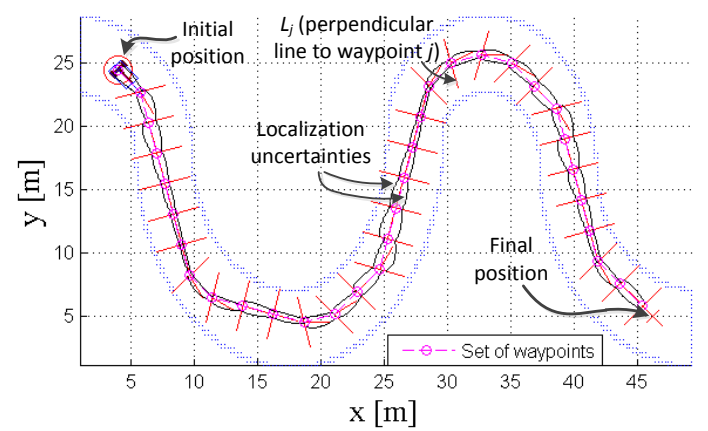

(a)

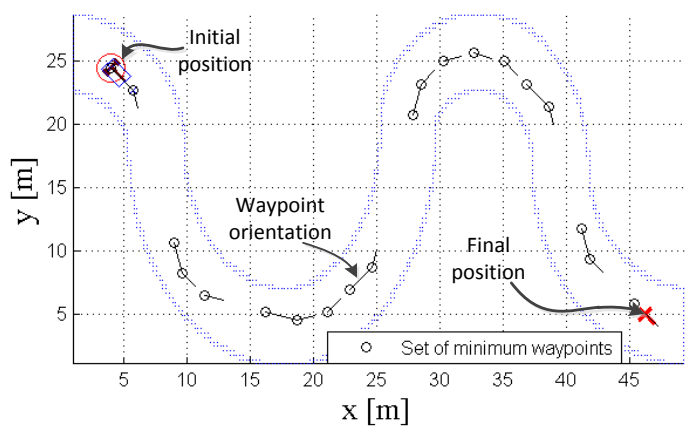

(b)

Fig. 14 a) Set of obtained waypoints using Algorithm 3 based on expanding tree and b) Minimum set of waypoints obtained by Algorithm 5 .

timal solution with minimal computational and memory requirements [15]. Moreover, $\mathrm{RRT}^{*}$ is a samplingbased algorithm and the optimal solution depends on the number of iterations of the RRT* algorithm, i.e., more is the number of iterations (more samples in the C-space free $_{\text {) }}$ closer is the obtained solution to the effective optimal global solution. Therefore, to compare the solutions obtained by the OMWS-ET with those obtained by the RRT* some little modifications in Algorithm 3 were made. The line 6 of Algorithm 3 was changed by a for loop from 0 to the maximum iteration number and the selection of the final pose at each iteration is obtained by the sampling in $\mathrm{C}_{- \text {space }_{\text {free }}}$ (qrandom) as the RRT* Algorithm [15]. It is to be noted that $q_{\text {random }}$ corresponds to a random sample (position) from a uniform distribution in the $\mathrm{C}$-space free $_{\text {. }}$

To compare the two algorithms (OMWS-ET and RRT $^{*}$ ), the safest obtained solution (wich maximizes the distance to the border) is used as criterion. Therefore, the parameters of the cost function of OMWS-ET (18) are fixed to: $k_{1}=1.0, k_{2}=k_{3}=k_{4}=0.0$ and $k_{h}=0.1$. In addition, the other parameters are fixed as: the branch number $n_{t}=5$; the edge distance $\xi=2.5 \mathrm{~m}$ and $\Delta \alpha=15^{\circ}$. The RRT ${ }^{*}$ algorithm described in [15] was also modified to obtain a cost function according to the safety $\bar{w}_{i}$ (distance to the border) instead of an Euclidean distance between nodes. The kinematic model (5) with constant linear velocity and steering angles $\left(v=1.0 \mathrm{~m} / \mathrm{s}\right.$ and $\left.\gamma=-15,-7.5,0,7.5,15^{\circ}\right)$ respectively, during $t_{\text {exp }}=2.5 \mathrm{~s}$ was used to produce the new nodes of the RRT* (cf. Section 1.2). The maximum number of iteration for both algorithm is fixed to $n_{I}=5000$.

Fig. 15 shows the obtained path solutions according to RRT*, OMWS-ET and also to Voronoï [20] algorithms. The Voronoï obtained path (cf. Fig. 15(c)) is given only because it is the best reference w.r.t. the adopted comparison criterion (safety criterion). Indeed, Voronoï path permits always to obtain the safest possible path [20] It can be noted that the two obtained path using RRT* and OMWS-ET are generally enough close and far from the way border (cf. Fig. 15(a) and 15(b)). Important differences are nevertheless observed in the obtained final results (cf. Fig. 15(c)). In fact, 


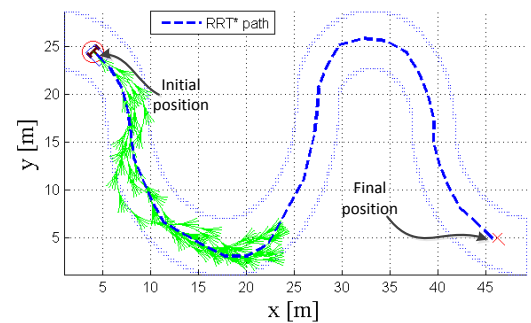

(a) $\mathrm{RRT}^{*}$ with a few branches

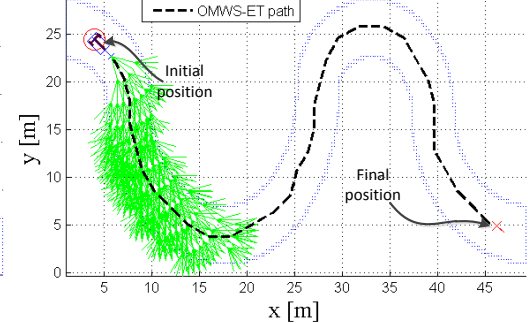

(b) OMWS-ET with a few branches

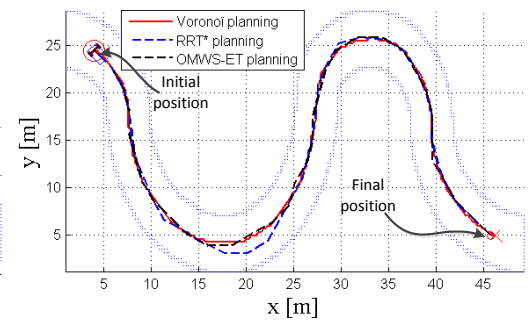

(c) Comparison with Voronoï

Fig. 15 Three obtained path according to Voronoï, RRT* and OMWS-ET.

the obtained set of waypoint using RRT* are closer to the border which is due to the fact that RRT* expands its branches while adopting constant commands $\left(v, \gamma, t_{\text {exp }}\right)$. These constant commands generate the next nodes with only a single possible orientation (for each node). Contrary to that, in the proposed OMWSET, each new obtained node $q_{j}$ has different possible orientations and velocities, thus, for the same position, much more possible vehicle's states (different orientations and velocity set-points) are taking into account in the optimization process.

Table 2 shows, as in the last subsection, different performance criteria to compare the obtained path. It is shown that the obtained path based on OMWS-ET is closer than the RRT* to the optimal obtained solution using Voronoï methodology. It validates that the proposed OMWS-ET is more efficient than the RRT*, in the sens that it explores much more possibilities in the vehicle/environment/task state space.

It is important to mention also, that the proposed OMWS-ET methodology is related to the adopted navigation strategy (cf. Section 2), which uses set-points based on suitable static/dynamic waypoints instead of trajectory tracking methods. OMWS-ET method takes into account the vehicle's kinematics constraints and uncertainites as well as the used control law (cf. subsection 3.2). RRT* method is more suitable for navigation strategies based on trajectory following [15].

\subsubsection{Specific scenario cases}

We show in what follows other minimum set of obtained waypoints for different scenarios using the method based on expanding tree (Algorithm 3). Fig. 16(a)

\begin{tabular}{|c|c|c|}
\cline { 2 - 3 } \multicolumn{1}{c|}{} & length $[\mathrm{m}]$ & $d_{\text {border }}[\mathrm{m}]$ \\
\hline Voronö̈ & 86.00 & 69.2931 \\
\hline RRT $^{*}$ & 83.42 & 62.1736 \\
\hline OMWS-ET & 82.50 & 65.5926 \\
\hline
\end{tabular}

Table 2 Comparison between Voronoï, RRT* and OMWSET. shows the set of waypoints while considering the edge distance $\xi=10 \mathrm{~m}$ with an objective to obtain the fastest trajectory from the initial to the final positions, while not colliding with the road limits. The constant values are $k_{1}=0.1, k_{2}=0.7, k_{3}=0.1, k_{4}=0.1$ and $k_{h}=0.1$. The minimum set of waypoints allows the vehicle to generate a minimum time trajectory as in [26]. This trajectory has a segment close to the route boundaries (tangent to the borders) which allows to navigate applying the maximum velocity.

Fig. 16(b) shows the use of the proposed OMWSET for the specific case where a reference path already exists for the navigation of the vehicle. In this case, the set of waypoints will be chosen as close as possible to the considered path (depends on the chosen values of $\xi$ and $\Delta \alpha$ in Algorithm 3). The set of waypoints obtained using OMWS-ET allows thus more flexible and safe navigation of the vehicle between the waypoints (cf. the criterion to optimize in (18)). The edge distance $\xi$ is set to $1 \mathrm{~m}$. The minimum set of waypoints are obtained while considering the term $\bar{w}_{j}(18)$ as the normalized minimum distance of the node $q_{j}$ to the reference path. The constant values are set to $k_{1}=0.6$, $k_{2}=0.2, k_{3}=0.1, k_{4}=0.1$ and $k_{h}=0.1$. The values of $\xi$ and $\Delta \alpha$ can produce some waypoints outside the reference trajectory, e.g., if we decrease the values of $\xi$ and $\Delta \alpha$ and increase the number of branches $n_{t}$ then the waypoints will be on the reference trajectory. In [33], the waypoints are selected while considering only the points in the reference trajectory. It consists on analyzing the orientation variation of each points on the trajectory. In our case, the waypoints are selected in the environment to be close to the reference trajectory which allows to obtain less number of waypoints than the method used in [33].

\subsubsection{Deterministic versus probabilistic}

This simulation shows the comparison between a deterministic and probabilistic expanding tree (i.e., where the values of $\xi$ and $\alpha$ are probabilistically taken from 


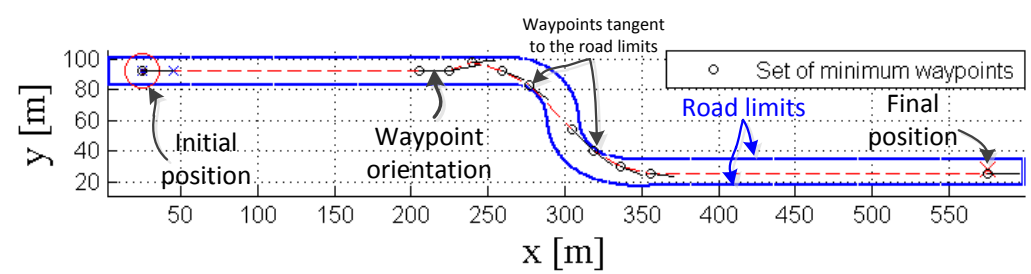

(a) Minimum set of waypoints for fastest trajectory.

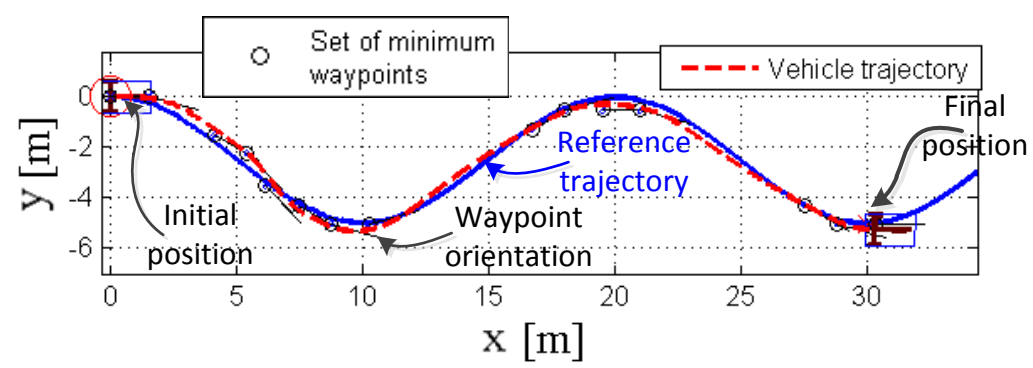

(b) Minimum set of waypoints in a reference path.

Fig. 16 Different scenario for OMWS-ET.

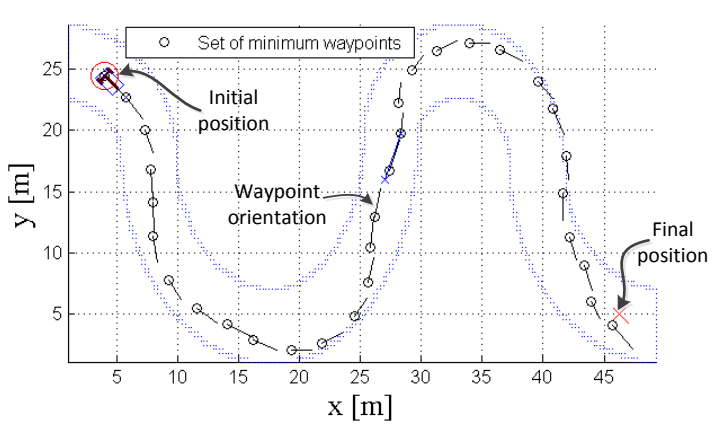

Fig. 17 Set of waypoints using probabilistic expanding tree.

an interval, instead of, to be fixed by the designer). Fig. 17 shows the minimum set of waypoints obtained using probabilistic expanding tree, where $\xi \in[0,2.5]$ and $\alpha \in\left[-30^{\circ}, 30^{\circ}\right]$. The constant values are $k_{1}=0.6$, $k_{2}=0.2, k_{3}=0.1, k_{4}=0.1$ and $k_{h}=0.1$. The processing time of the method with probabilistic expanding tree is less than the method with deterministic expanding tree. Nevertheless, the set of waypoints are not the optimal solution. The advantages of probabilistic selection of $\xi$ and $\alpha$ is to reduce the convergence time and to obtain an online implementation [18], [32]. In future works, the choice of the variation of $\xi$ and $\alpha$ will be oriented to improve the efficiency of the algorithm.

\subsubsection{Extension to multi-robot formation}

Our method based on expanding tree (Algorithm 3) was extended to multi-robot formation where the formation is defined only according to the leader configuration [9] (cf. Fig. 18). As mentioned before, the OMWS-ET algorithm takes into account the vehicle model. To cope with this multi-robot task, it is sufficient to adapt the term $\Delta \bar{e}_{l_{i} j}(24)$ in order to consider all trajectories of the group of UGVs. Fig. 19 shows the minimum set of waypoints for a line formation $\left(d_{i}=6 \mathrm{~m}\right.$ and $\left.\phi_{i}=180^{\circ}\right)$ with two vehicles. The constant values are the same as the last simulation. The set of waypoints for the leader UGV are close to the curve road boundaries because the formation needs enough space to turn while keeping the rigid formation shape. The follower (blue square) is always inside of the road boundaries.

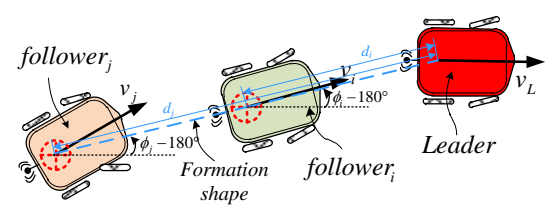

Fig. 18 Multi-robot formation (straight line shape).

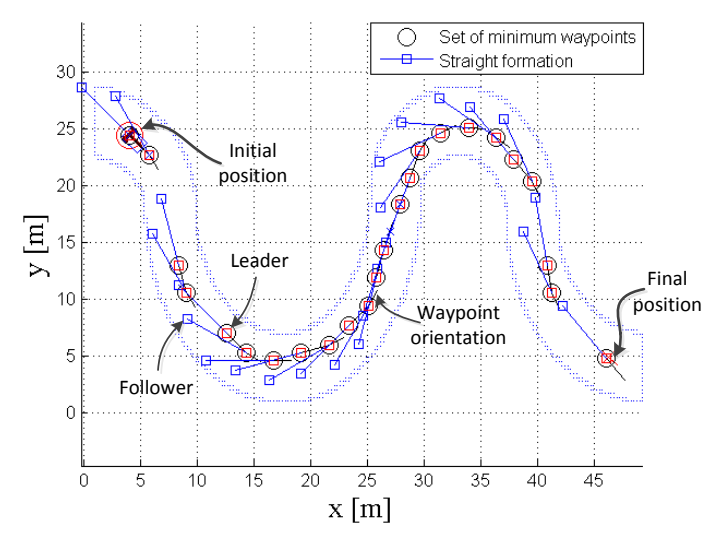

Fig. 19 Minimum set of waypoints for multi-robot formation obtained by Algorithm 3 based on expanding tree. 


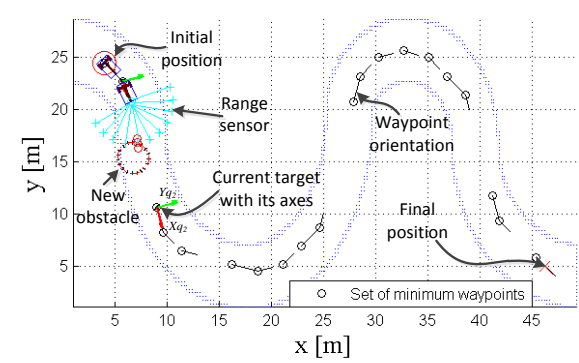

(a) Unexpected obstacle is detected.

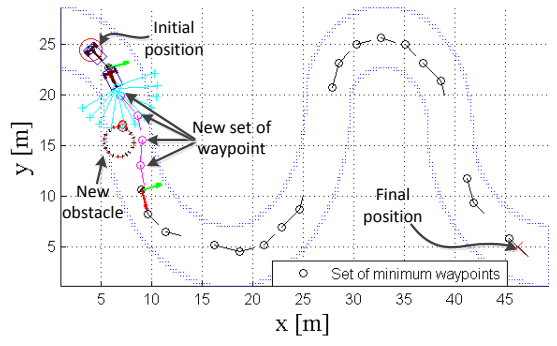

(b) Local replanning.

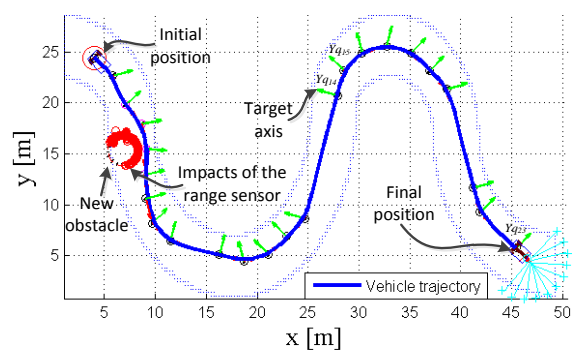

(c) Safe vehicle trajectory.

Fig. 21 Local replanning for unexpected obstacle.

\subsubsection{Local replanning for unexpected obstacles}

The proposed method OMWS-ET is adapted to local replanning when an unexpected static obstacle is detected in the environment. Fig. 20 shows the used architecture to activate the replanning of the vehicle's movements based on an initial set of waypoints already obtained using OMWS-ET. The vehicle starts the navigation through the successive waypoints (cf. subsection 2.1) from the initial set of waypoints. They were already computed using the OMWS-ET in the known environment (cf. subsection 3.2). The vehicle uses a range sensor to detect any unforeseen obstacle (cf. Fig. 21(a)). A local replanning is activated when any new obstacle is detected. This replanning takes into account the current environment state, the current vehicle pose and the current waypoint to obtain a new local set of waypoints (cf. Fig. 21(b)). If the current waypoint is unreachable (due to the presence of the obstacle) then the final position is replaced by the next waypoint in the list and so on. If no solution is found then the vehicle will stop in its current pose. Figure 21(b) shows an example of the local replanning using the set of waypoints given

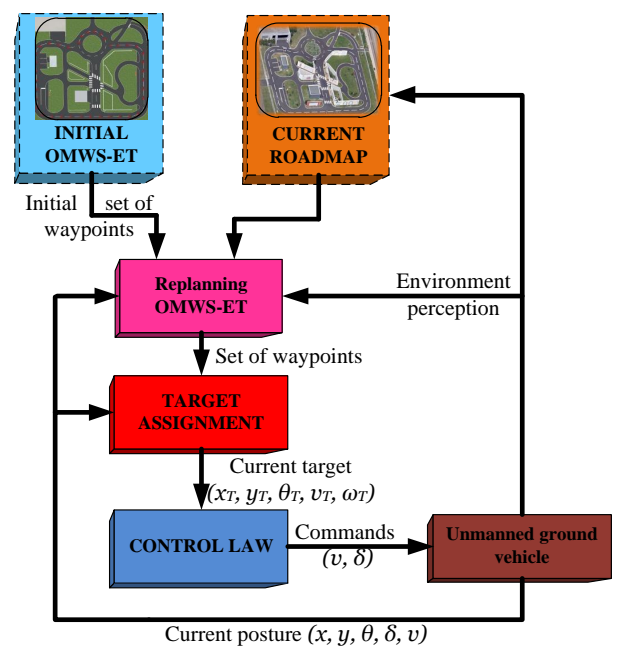

Fig. 20 Schema of the local replanning. in subsection 4.1.1 (as initial set of waypoints) (cf. Fig. 14(b)). Finally, the vehicle moves through the new set of waypoints while guaranteeing a safe navigation (cf. Fig. 21(c)).

\subsection{Experimental results}

The navigation strategy and the proposed method based on expanding tree was also experimented with the actual VIPALAB vehicle (cf. Fig. 22) in an experimental environment named PAVIN (Plate-forme d'Auvergne pour Véhicules INtelligents) (cf. Fig 23). These experiments can be found online ${ }^{1}$. This vehicle carry different embedded proprioceptive and exteroceptive sensors such as odometers, gyrometer, steering angle sensor and an RTK-GPS. Each vehicle uses a combination of RTKGPS and gyrometer to estimate its current position and orientation at a sample time of $T_{s}=0.01 \mathrm{~s}$.

\footnotetext{
1 http://maccs.univ-bpclermont.fr/uploads/Profiles/VilcaJM/OMWS.mp4
}

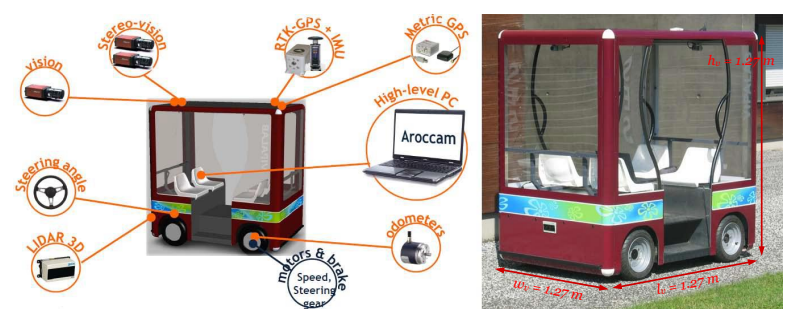

Fig. 22 VIPALAB electric urban vehicle.

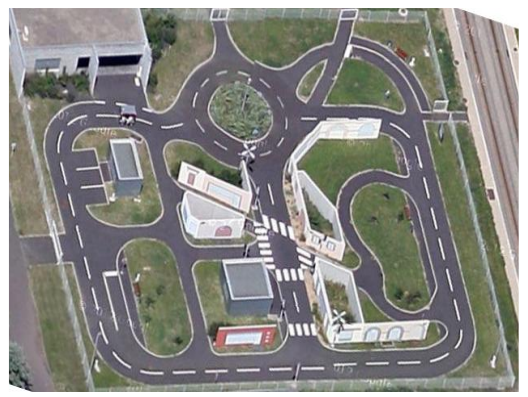

Fig. 23 PAVIN experimental platform (Clermont-Ferrand, France). 


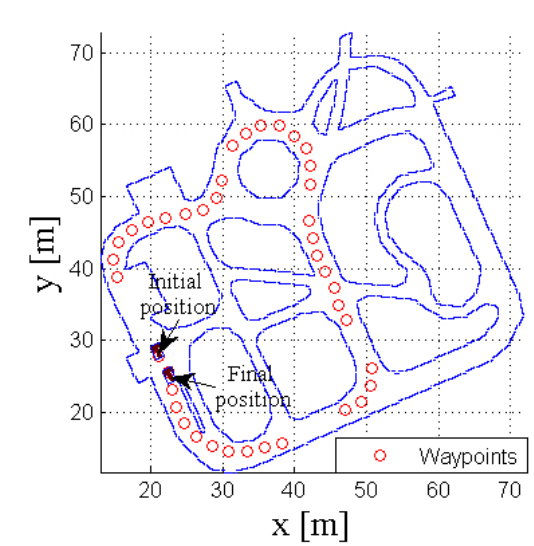

(a) First experiment: Safe planning.

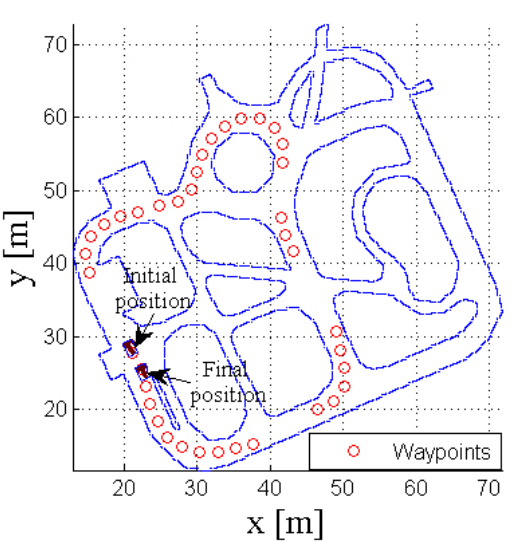

(b) Second experiment: Steering angle (c) Comparison between experiments. minimization.

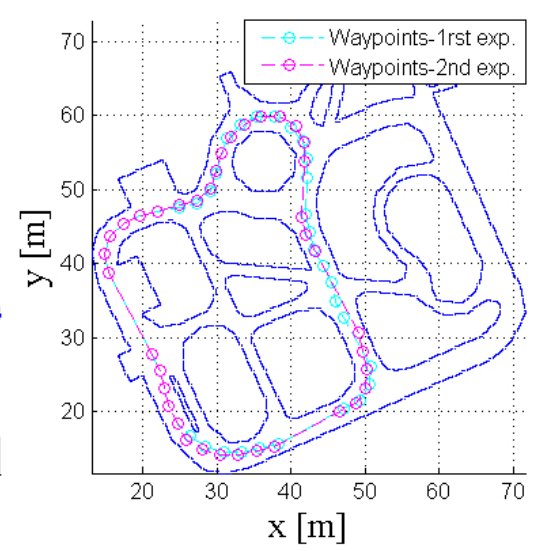

Fig. 24 Different set of obtained waypoints.

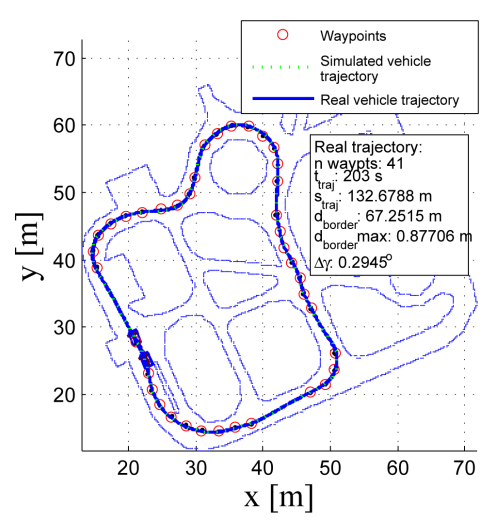

(a) First experiment.

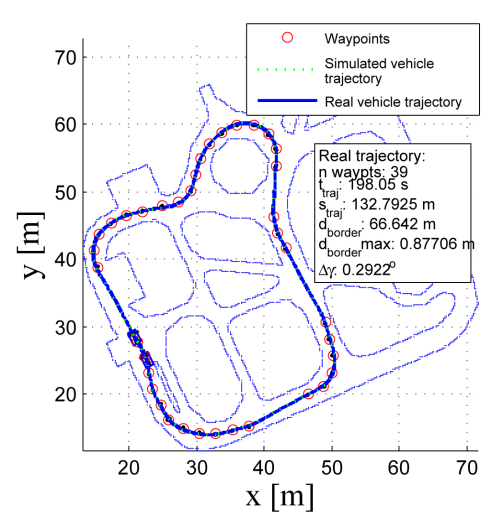

(b) Second experiment.

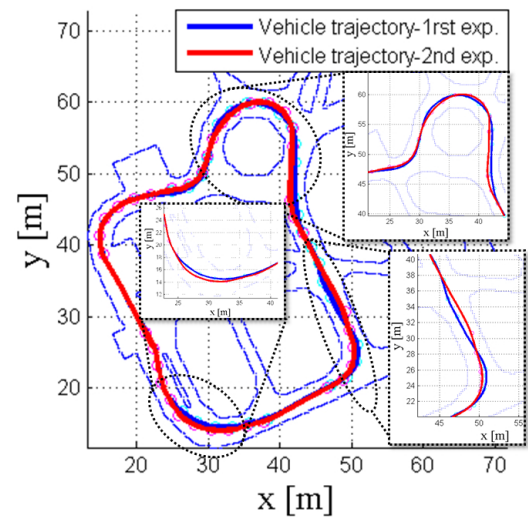

(c) Comparison between actual experiments.

Fig. 25 Actual vehicle's trajectories for different obtained set of waypoints.

A metric map of the environment PAVIN [12] is used by the proposed method (Algorithm 3). This map allows to implement the navigation through successive waypoints in a real vehicle (cf. Section 2.1). The proposed method based on expanding tree computes the set of geo-referenced waypoints with optimal configuration. Certain areas are restricted to guide the Algorithm 3 through PAVIN platform which has intersections and roundabout (cf. Fig. 24). In our case, these restricted areas were selected by the user, nevertheless the selection can be made by considering the topological map of the environment. We experiment the proposed OMWSET to make a comparison between two cases: the first, corresponds to give more priority for the safety criteria in (18) and the second gives more priority for the minimum angle steering rate. The analysis of the obtained solutions will be given in what follows. Moreover, the actual vehicle's trajectories are compared for these different set of waypoints.

Fig. 24 and 25 show respectively the minimum obtained set of waypoints and the corresponding vehicle's trajectories (in simulation and actual experiment). Fig. 24(a) shows the set of waypoints of the first experiment where the constant values of the cost function (18) are $k_{1}=0.6, k_{2}=0.2, k_{3}=0.1, k_{4}=0.1$ and $k_{h}=0.4$. The safety $\left(k_{1}\right)$ has the highest priority in this experiment. Therefore, these waypoints guide the vehicle to be close to the middle of the route (cf. Fig. 25(a)). Fig. 24(b) shows the set of waypoints of the second experiment where the constant values are $k_{1}=0.3, k_{2}=0.2$, $k_{3}=0.4, k_{4}=0.1$ and $k_{h}=0.4$. The minimal steering angle rate $k_{3}$ has the highest priority in this experiment. The obtained result shows that the obtained waypoints are localized very close to the border of the road (cf. Fig. 25(b)). Fig. 25(a) and 25(b) show the simulated and the actual vehicle trajectories. It can be observed that they are very close (maximal error between them is less than $0.15 \mathrm{~m}$ ). We can conclude thus that the proposed optimal multi-criteria waypoint selection based on Expanding Tree (OMWS-ET, performed off-line (cf. Section 3.2)) permits to cope accurately with actual environment and experiments. 

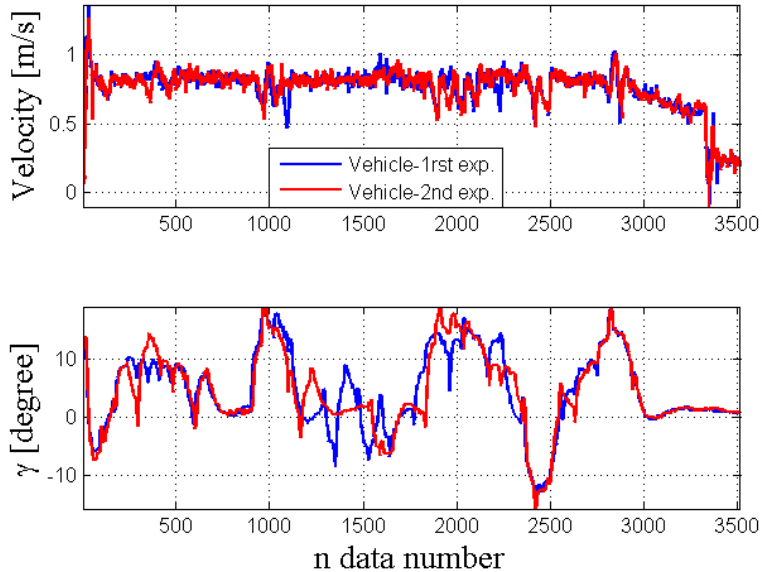

Fig. 26 Vehicle velocities and steering angles progress for each set of obtained waypoints.

Fig. 24(c) and 25(c) show the comparison between the set of waypoints and the real trajectories of both experiments. The velocities and steering angle of the vehicle while tracking each waypoint are shown in Fig. 26. This figure shows the values with noise due to the encoder inaccuracies.

Table 3 shows different performance criteria to compare the set of waypoints where: $n_{w}$ is the number of waypoints, $T$ is the navigation time, $l_{U G V}$ is the traveled distance, $d_{\text {border }}$ is the sum of minimum distance to the road boundaries and $\Delta \gamma$ is the root mean square (rms) of the steering angle rate. We note that the first experiment has $n_{w}$ greater than the second experiment. It is due to the fact that the first experiment has the safety as a priority. The proposed Algorithm 3 selects thus more waypoints to allows the vehicle to navigate as farther as possible from the road borders. It can be noticed by $d_{\text {border }}$ where its value is bigger in the first experiment than the second. Furthermore, the values of $\Delta \gamma$ is less in the second experiment because the highest priority was for the steering angle rate. Therefore, the vehicle can navigate with higher velocity along the trajectory and the navigation time is smaller than the first experiment.

\begin{tabular}{|l|l|c|c|c|c|c|}
\cline { 3 - 7 } \multicolumn{2}{c|}{} & $n_{w}$ & $T[s]$ & $l_{U G V}[\mathrm{~m}]$ & $d_{\text {border }}[\mathrm{m}]$ & $\Delta \gamma\left[^{\circ}\right]$ \\
\hline 1rst & Sim. & 41 & 200 & 132.81 & 67.35 & 0.3123 \\
\cline { 2 - 7 } exp. & Real & 41 & 203 & 132.68 & 67.25 & 0.2945 \\
\hline 2nd & Sim. & 39 & 199 & 133.00 & 66.54 & 0.3089 \\
\cline { 2 - 7 } exp. & Real & 39 & 198 & 132.79 & 66.64 & 0.2922 \\
\hline
\end{tabular}

Table 3 Comparison among the set of waypoints

\section{Conclusion}

This paper has presented two planning methods to obtain the optimal waypoints configuration (Optimal Multicriteria Waypoints Selection based on Expanding Tree (OMWS-ET) and Grid Map (OMWS-GM)) which guarantees safe, smooth and feasible vehicle navigation in a structured environment. The flexible navigation strategy throughout optimal and discrete selected waypoints was also presented. It allows to avoid any trajectory planning which could be time consuming. The proposed OMWS-GM is based on the $\mathrm{A}^{*}$ algorithm with an additional term to consider the orientation change between successive cells. OMWS-ET uses a multi-criteria function which takes into account the vehicle model and uncertainties to obtain the optimal set of waypoints configurations (position, orientation and velocity). Moreover, it has been shown that the proposed OMWS-ET is much more accurate and flexible than OMWS-GM. A multitude of simulations and experimental results demonstrate the efficiency and reliability of the proposed OMWS-ET in different cases (trajectory specification, deterministic versus probabilistic, comparison with RRT*, multi-robot task, local replanning according to the multi-criteria optimization).

In future works, an extension using the dynamic model of the vehicle and $3 D$ position will be developed (notably for unmanned aerial vehicle). The enhancement of the proposed methods for hard real time application will be developed. Genetic algorithm will be notably investigated. In addition, we will extend the proposed strategies for robust navigation in formation of a group of robots.

\section{Appendix}

This section described briefly the stability analysis based on Lyapunov method used to demonstrate the convergence of the vehicle to the target posture, i.e., for a finite time, the error system $\left(e_{x}, e_{y}, e_{\theta}\right)$ converges to zero [16]. Let us first define the Lyapunov function $V$ by (26). It is a function of three parameters which depend on: the distance $d$ between the target and vehicle positions, the distance $d_{l}$ from the vehicle to the target line (line that pass through the target position with orientation equal to the target orientation), this term is related to the Line of Sight and Flight of the target, and the orientation error $e_{\theta}$ between the vehicle and the target (cf. Fig. 5). It is represented by:

$$
\begin{aligned}
V & =\frac{1}{2} K_{d} d^{2}+\frac{1}{2} K_{l} d_{l}^{2}+K_{o}\left[1-\cos \left(e_{\theta}\right)\right] \\
& =\frac{1}{2} K_{d} d^{2}+\frac{1}{2} K_{l} d^{2} \sin ^{2}\left(e_{R T}\right)+K_{o}\left[1-\cos \left(e_{\theta}\right)\right]
\end{aligned}
$$


where the initial values of $e_{R T}$ and $e_{\theta}$ satisfy:

$\left.e_{R T} \in\right]-\pi / 2, \pi / 2\left[\right.$ and $\left.e_{\theta} \in\right]-\pi / 2, \pi / 2[$

These conditions (27) guarantee that the target is ahead to the vehicle w.r.t. its orientation. Moreover, (27) has open interval that allows to avoid local minimum. Therefore, $V$ is a positive-definite function [16].

The Lyapunov function (26) can be written according to $e_{x}, e_{y}$ as follows:

$V=\frac{1}{2}\left(e_{x}^{2}+e_{y}^{2}\right)\left[K_{d}+K_{l} \sin ^{2}\left(e_{R T}\right)\right]+K_{o}\left[1-\cos \left(e_{\theta}\right)\right]$

To guarantee the system stability, $\dot{V}$ has to be negativedefinite [16]. By taking the derivate of (28), (2) and (4) and using $(7)$ and $(8), \dot{V}$ can be written:

$$
\begin{aligned}
\dot{V}= & \left(e_{x} \dot{e}_{x}+e_{y} \dot{e}_{y}\right)\left[K_{d}+K_{l} \sin ^{2}\left(e_{R T}\right)\right] \\
& +K_{l} d^{2} \sin \left(e_{R T}\right) \cos \left(e_{R T}\right) \dot{e}_{R T}+K_{o} \sin \left(e_{\theta}\right) \dot{e}_{\theta} \\
= & {\left[-e_{x} v_{b}+v_{T} e_{y} \sin \left(e_{\theta}\right)\right]\left[K_{d}+K_{l} \sin ^{2}\left(e_{R T}\right)\right] } \\
+ & K_{l} \sin \left(e_{R T}\right) \cos \left(e_{R T}\right)\left[\frac{d^{2} v_{T}}{r_{c_{T}}}-v_{T} e_{x} \sin \left(e_{\theta}\right)-e_{y} v_{b}\right] \\
+ & K_{o} \sin \left(e_{\theta}\right)\left(\frac{v_{T}}{r_{c_{T}}}-v_{T} \cos \left(e_{\theta}\right) c_{c}-v_{b} c_{c}\right)
\end{aligned}
$$

Using (6) in the first two terms of (29) and factorizing the common terms, it holds that:

$$
\begin{aligned}
\dot{V}= & v_{T} \sin \left(e_{\theta}\right)\left[K_{d} e_{y}-K_{l} d \sin \left(e_{R T}\right) \cos \left(e_{\theta}\right)\right] \\
& +\frac{v_{T}}{r_{c_{T}}}\left[d^{2} K_{l} \sin \left(e_{R T}\right) \cos \left(e_{R T}\right)+K_{o} \sin \left(e_{\theta}\right)\right] \\
& -v_{b}\left[K_{d} e_{x}+K_{l} d \sin \left(e_{R T}\right) \sin \left(e_{\theta}\right)+K_{o} \sin \left(e_{\theta}\right) c_{c}\right] \\
& -v_{T} K_{o} \sin \left(e_{\theta}\right) \cos \left(e_{\theta}\right) c_{c}
\end{aligned}
$$

Finally, using (9) and (10) in (30), we obtain:

$$
\begin{aligned}
\dot{V}= & -K_{x}\left[K_{d} e_{x}+K_{l} d \sin \left(e_{R T}\right) \sin \left(e_{\theta}\right)+K_{o} \sin \left(e_{\theta}\right) c_{c}\right]^{2} \\
& -v_{T} K_{o} K_{\theta} \sin ^{2}\left(e_{\theta}\right)-v_{T} K_{o} K_{R T} \sin ^{2}\left(e_{R T}\right) \leq 0
\end{aligned}
$$

Eq. (31) shows that the system is stable while the initial conditions (27) are satisfied. To ensure the asymptotic stability of the error system, $\dot{V}$ has to be a negativedefinite function. Let us exhibit the case where $\dot{V}=0$ with $v_{T}>0$ and $v_{T}=0$. Firstly, when $v_{T}>0$ and using the initial assumption $\mathbf{K}>0$, it is straightforward to show that $e_{x}, e_{\theta}, e_{R T}$ are equal to zero to satisfy (31), then according to (5), (4) and (27) $d$ is equal to zero $\left(e_{y}=0\right)$. Hence, $\dot{V}$ is equal to zero when $v_{T}>0$, only if $\left(e_{x}, e_{y}, e_{\theta}\right)=(0,0,0)$.

Secondly, let us consider the case where $v_{T}=0$. The initial assumption is identical. Hence, the second and third terms of (31) are equal to zero when $v_{T}=0$.
Additionally, when $v_{T}=0$, we consider that $r_{c_{T}} \rightarrow \infty$, consequently the first term of $\dot{V}$ is equal to zero when:

$K_{d} e_{x}+K_{l} d \sin \left(e_{R T}\right) \sin \left(e_{\theta}\right)+K_{o} \sin \left(e_{\theta}\right) c_{c}=0$

Replacing (10) with $r_{c_{T}} \rightarrow \infty$ in (32), the following expression is obtained:

$$
\begin{aligned}
0= & K_{d} e_{x}+K_{l} d \sin \left(e_{R T}\right) \sin \left(e_{\theta}\right) \\
& +\tan \left(e_{\theta}\right)\left[K_{d} e_{y}-K_{l} d \sin \left(e_{R T}\right) \cos \left(e_{\theta}\right)\right] \\
& +K_{o} \sin \left(e_{\theta}\right)\left[K_{\theta} \tan \left(e_{\theta}\right)+\frac{K_{R T} \sin ^{2}\left(e_{R T}\right)}{\sin \left(e_{\theta}\right) \cos \left(e_{\theta}\right)}\right] \\
= & K_{d}\left[e_{x}+e_{y} \tan \left(e_{\theta}\right)\right]+K_{o} K_{\theta} \frac{\sin ^{2}\left(e_{\theta}\right)}{\cos \left(e_{\theta}\right)} \\
& +K_{o} K_{R T} \frac{\sin ^{2}\left(e_{R T}\right)}{\cos \left(e_{\theta}\right)}
\end{aligned}
$$

Using (6) in (33), we obtain:

$K_{d} d \frac{\cos \left(e_{R T}\right)}{\cos \left(e_{\theta}\right)}+K_{o} K_{\theta} \frac{\sin ^{2}\left(e_{\theta}\right)}{\cos \left(e_{\theta}\right)}+K_{o} K_{R T} \frac{\sin ^{2}\left(e_{R T}\right)}{\cos \left(e_{\theta}\right)}=0$

Eq. (34) exhibits quadratic terms. Consequently, considering the initial conditions $(27), \cos \left(e_{R T}\right)$ and $\cos \left(e_{\theta}\right)$ are greater than zero. Therefore, all the terms of (34) are positive and they must be equal to zero, i.e., $d=$ $e_{\theta}=e_{R T}=0$, and if $d=0$ then $e_{x}, e_{y}=0$. Hence, from (34), $\dot{V}$ is equal to zero when $v_{T}=0$ and $r_{c_{T}} \rightarrow \infty$, only if $\left(e_{x}, e_{y}, e_{\theta}\right)=(0,0,0)$.

Conclusively, if $v_{T}>0$ and $v_{T}=0, V$ is always strictly positive and $\dot{V}$ is always strictly negative while $\left(e_{x}, e_{y}, e_{\theta}\right) \neq(0,0,0)$. Therefore, the errors system is asymptotically stable while the initial vehicle conditions (27) are satisfied.

\section{References}

1. Abbadi, A., Matousek, R., Petr Minar, P.S.: RRTs review and options. In: International Conference on Energy, Environment, Economics, Devices, Systems, Communications, Computers (2011)

2. Adouane, L., Benzerrouk, A., Martinet, P.: Mobile robot navigation in cluttered environment using reactive elliptic trajectories. In: 18th IFAC World Congress (2011)

3. Aicardi, M., Casalino, G., Bicchi, A., Balestrino, A.: Closed loop steering of unicycle like vehicles via lyapunov techniques. Robotics Automation Magazine, IEEE 2(1), 27-35 (1995)

4. Bellman, R.: A markovian decision process. Journal of Mathematics and Mechanics 6, 679 - 684 (1957)

5. Bertsekas, D.P.: Dynamic Programming and Optimal Control, vol. I. Athena Scientific (1995)

6. Bonfè, M., Secchi, C., Scioni, E.: Online trajectory generation for mobile robots with kinodynamic constraints and embedded control systems. In: 10th International IFAC Symposium on Robot Control. Croatia (2012) 
7. Choset, H., Lynch, K.M., Hutchinson, S., Kantor, G., Burgard, W., Kavraki, L.E., Thrun, S.: Principles of Robot Motion: Theory, Algorithms, and Implementation. MIT Press (2005)

8. Connors, J., Elkaim, G.H.: Manipulating b-spline based paths for obstacle avoidance in autonomous ground vehicles. In: ION National Technical Meeting, ION NTM 2007. San Diego, CA, USA (2007)

9. Consolini, L., Morbidi, F., Prattichizzo, D., Tosques, M.: Leader-follower formation control of nonholonomic mobile robots with input constraints. Automatica 44(5), 1343 - 1349 (2008)

10. Gu, T., Dolan, J.M.: On-road motion planning for autonomous vehicles. In: C.Y. Su, S. Rakheja, H. Liu (eds.) Intelligent Robotics and Applications, vol. 7508. Springer Berlin Heidelberg (2012)

11. Horst, J., Barbera, A.: Trajectory generation for an onroad autonomous vehicle. Proceedings of the SPIE: Unmanned Systems Technology VIII (2006)

12. The Institut Pascal Data Sets: (2013). http://ipds.univ-bpclermont.fr.

13. Jazar, R. N.: Vehicle Dynamics: Theory and Application, Chapter 7. Springer-Verlag (2014)

14. Kallem, V., Komoroski, A., Kumar, V.: Sequential composition for navigating a nonholonomic cart in the presence of obstacles. IEEE Transactions on Robotics 27(6), 1152 - 1159 (2011)

15. Karaman, S. and Frazzoli, E.: Sampling-based Algorithms for Optimal Motion Planning. International Journal of Robotics Research 30(7), 846 - 894 (2011)

16. Khalil, H. K.: Nonlinear Systems. Prentice Hall (2002) (1986)

17. Khatib, O.: Real-time obstacle avoidance for manipulators and mobile robots. The International Journal of Robotics Research 5, pp.90-99 (1986)

18. Kuwata, Y., Fiore, G.A., Teo, J., Frazzoli, E., How, J.P.: Motion planning for urban driving using rrt. In: International Conference on Intelligent Robots and Systems, pp. $1681-1686$ (2008)

19. Labakhua, L., Nunes, U., Rodrigues, R., Leite, F.: Smooth trajectory planning for fully automated passengers vehicles: Spline and clothoid based methods and its simulation. In: J. Cetto, J.L. Ferrier, J.M. Costa dias Pereira, J. Filipe (eds.) Informatics in Control Automation and Robotics, Lecture Notes Electrical Engineering, vol. 15, pp. 169-182. Springer Berlin Heidelberg (2008)

20. Latombe, J.C.: Robot Motion Planning. Kluwer Academic Publishers, Boston, MA (1991)

21. LaValle, S.M.: Planning Algorithms. Cambridge Univ. Press (2006)

22. Lee, J.W., Litkouhi, B.: A unified framework of the automated lane centering/changing control for motion smoothness adaptation. In: 15th International IEEE Conference on Intelligent Transportation Systems (ITSC), pp. 282-287 (2012)

23. Luca, A.D., Oriolo, G., Samson, C.: Feedback control of a nonholonomic car-like robot. In: J.P. Laumond (ed.) Robot Motion Planning and Control, pp. 171-253. Springer-Verlag, Berlin (1998)

24. Maalouf, E., Saad, M., Saliah, H.: A higher level path tracking controller for a four-wheel differentially steered mobile robot. Robotics and Autonomous Systems 54, 23 $-33(2006)$

25. Martins, M.M., Santos, C.P., Frizera-Neto, A., Ceres, R.: Assistive mobility devices focusing on smart walkers: Classification and review. Robotics and Autonomous Systems 60(4), $548-562(2012)$
26. Rucco, A., Notarstefano, G., Hauser, J.: Computing minimum lap-time trajectories for a single-track car with load transfer. In: Decision and Control (CDC), 2012 IEEE 51st Annual Conference on, pp. 6321-6326 (2012)

27. Sezen, B.: Modeling automated guided vehicle systems in material handling. Otomatiklestirilmi Rehberli Arac Sistemlerinin Transport Tekniginde Modellemesi, Dou Universitesi Dergisi, 4(2), 207 - 216 (2011)

28. Sharma, S., Taylor, M.E.: Autonomous waypoint selection for navigation and path planning: A navigation framework for multiple planning algorithms. Tech. rep. (2012)

29. Siciliano, B., Khatib, O. (eds.): Springer Handbook of Robotics, Part E-34. Springer (2008)

30. Stoeter, S.A., Rybski, P.E., Stubbs, K.N., McMillen, C.P., Gini, M., Hougen, D.F., Papanikolopoulos, N.: A robot team for surveillance tasks: Design and architecture. Robotics and Autonomous Systems 40(2-3), 173 $183(2002)$

31. Szczerba, R., Galkowski, P., Glicktein, I., Ternullo, N.: Robust algorithm for real-time route planning. IEEE Transactions on Aerospace and Electronic Systems 36(3), 869-878 (2000)

32. Vaz, D.A., Inoue, R.S., Grassi Jr., V.: Kinodynamic motion planning of a skid-steering mobile robot using rrts. In: Proceedings of the 2010 Latin American Robotics Symposium and Intelligent Robotics Meeting, LARS '10, pp. 73-78. IEEE Computer Society (2010)

33. Vilca, J., Adouane, L., Mezouar, Y., Lébraly, P.: An overall control strategy based on target reaching for the navigation of an urban electric vehicle. In: IEEE/RSJ International Conference on Intelligent Robots and Systems (IROS'13). Tokyo - Japan (2013)

34. Ziegler, J., Werling, M., Schroeder, J.: Navigating carlike robots in unstructured environment using an obstacle sensitive cost function. In: Proc. IEEE Intelligent Vehicle Sympsium (IV), pp. 787 - 791. Netherlands (2008) 